\title{
Asymmetric Catalytic Synthesis of $P$-Stereogenic Phosphines via a Nucleophilic Ruthenium Phosphido Complex
}

\author{
Vincent S. Chan, Ian C. Stewart, Robert G. Bergman, F. Dean Toste \\ Department of Chemistry, University of California, Berkeley, Berkeley, CA 94720
}

\section{Supporting Information}

Page \#

General Information

S-2

Materials

S-2

General Procedure for Asymmetric Alkylation

S-3

Synthetic Procedures

S-4

ORTEP Diagrams of $\mathbf{1}$ and $\mathbf{2}$

S-15

General Experimental Details for X-Ray Structure Determination

S-17

X-Ray Structural Parameters (Tables S-1 through S-14)

S-18

References

S-37 
General Information. Unless otherwise noted, reactions and manipulations were performed at ambient temperature in an inert atmosphere $\left(\mathrm{N}_{2}\right)$ glovebox, or using standard Schlenk and high vacuum line techniques. Glassware was oven-dried overnight at $150{ }^{\circ} \mathrm{C}$ or flame-dried under vacuum immediately prior to use. All NMR spectra were obtained at ambient temperature using Bruker DRX-500, AV-500, AVB-400, AVQ-400, or AV-300 spectrometers. ${ }^{1} \mathrm{H}$ NMR chemical shifts $(\delta)$ are reported in parts per million (ppm) relative to residual protiated solvent. Data are reported as follows: $(\mathrm{s}=$ singlet, $\mathrm{d}=$ doublet, $\mathrm{t}=$ triplet, $\mathrm{q}=$ quartet, $\mathrm{m}=$ multiplet; coupling constant(s) in $\mathrm{Hz}$; integration). ${ }^{13} \mathrm{C}$ NMR chemical shifts $(\delta)$ are reported in ppm relative to the carbon resonance of the deuterated solvent. Infrared (IR) spectra were recorded on a ThermoNicolet Avatar 370 as a thin film on a ZnSe crystal or as a Nujol mull between $\mathrm{NaCl}$ plates. Elemental analyses were performed at the University of California, Berkeley Microanalytical facility on a Perkin Elmer 2400 Series II CHNO/S Analyzer. X-ray structural analyses were performed by Dr. Fred Hollander and Dr. Allen Oliver in the University of California, Berkeley CHEXRAY facility. Enantiomeric excess was determined using a Shimatzu 10A VP Series Chiral HPLC.

Materials. Reagents were purchased from commercial suppliers, checked for purity and used without further purification unless otherwise noted. Liquids were degassed using three freeze-pump-thaw cycles and dried over 4A activated molecular sieves. Solids were stored in an inert $\left(\mathrm{N}_{2}\right)$ atmosphere glove box. Tetrahydrofuran, pentane, hexanes, toluene, diethyl ether and benzene (Fisher) were passed through a column of activated alumina (type A2, size 12 x 32, Purify Co.) under nitrogen pressure and sparged with $\mathrm{N}_{2}$ prior to use. Benzene- $d_{6}$ was dried over 4A sieves. THF- $d_{8}$ was vacuum transferred from purple sodium benzophenone/ketyl and stored under an inert atmosphere. $\quad(\mathrm{dmpe})_{2} \mathrm{Ru}(\mathrm{H})_{2}{ }^{1}, \quad\left[\mathrm{Ru}(\mathrm{H})\left(\mathrm{NH}_{2} \mathrm{NMe}_{2}\right)(\mathrm{cod})\right]\left[\mathrm{BPh}_{4}\right]^{2}, \quad$ and $\left(\mathrm{PPh}_{3}\right)_{3} \mathrm{Ru}(\mathrm{H})(\mathrm{Cl})^{3}$ were prepared according to literature procedures. 
General Procedure for Asymmetric Alkylation. In a nitrogen atmosphere glovebox, a one-dram vial was charged with ruthenium catalyst 10 (24 mg, $0.020 \mathrm{mmol}, 0.01$ equiv) and sodium tert-amyloxide ( $27 \mathrm{mg}, 0.24 \mathrm{mmol}, 1.2$ equiv), to which THF (0.8 mL) was added. Benzyl chloride ( $28 \mu \mathrm{L}, 0.24 \mathrm{mmol}, 1.2$ equiv) was syringed into the solution, which was then transferred into an NMR tube. The tube was fitted with a Cajon adapter and brought out of the glovebox. Using standard Schlenk techniques, the NMR tube was put under a positive pressure of nitrogen on a high vacuum manifold. The solution was cooled to $-78{ }^{\circ} \mathrm{C}$, and methylphenylphosphine (26 $\mu \mathrm{L}, 0.20 \mathrm{mmol}, 1.0$ equiv) was added directly to the reaction mixture using a Hamilton Gastight syringe. The NMR tube was then flame-sealed under an atmosphere of nitrogen. The contents were mixed by inverting the NMR tube, then warmed to $30{ }^{\circ} \mathrm{C}$. After $60 \mathrm{~h}$, the solution was warmed to room temperature and brought into the glovebox. The NMR tube was opened and the solution was transferred to a five-dram scintillation vial equipped with a stirbar. A $1.0 \mathrm{M}$ solution of $\mathrm{BH}_{3} \bullet \mathrm{THF}(2 \mathrm{~mL}, 2.0 \mathrm{mmol}, 10$ equiv) was added and the resulting solution was stirred for $30 \mathrm{~min}$ at room temperature. The following work-up was performed outside of the glovebox: Excess $\mathrm{BH}_{3}$ was quenched by slow addition of water; the aqueous solution was then extracted with ethyl acetate $(3 \times 10 \mathrm{~mL})$. The combined organic phase was washed with brine $(10 \mathrm{~mL})$, dried over $\mathrm{Na}_{2} \mathrm{SO}_{4}$, filtered and concentrated in vacuo. The crude product was purified by silica gel chromatography (conditions indicated below). 
trans-(dmpe $)_{2} \mathbf{R u}(\mathbf{H}) \mathbf{P M e P h}(\mathbf{1})$. In a nitrogen glove box a $50 \mathrm{~mL}$ glass-walled vessel equipped with a vacuum stopcock was charged with (dmpe) ${ }_{2} \mathrm{RuH}_{2}(0.50 \mathrm{~g}, 1.24 \mathrm{mmol}, 1.0$ equiv) and a stirbar. On a vacuum line using standard Schlenk techniques dry and degassed EtOH $(25 \mathrm{~mL})$ was added via cannula transfer. Methylphenylphosphine $(0.15 \mathrm{~mL}, 1.24 \mathrm{mmol}$, 1.0 equiv) was then added via syringe. After $3 \mathrm{~h}$, the volatile materials were removed in vacuo overnight, yielding the product as a bright orange solid $(0.66 \mathrm{~g}, 100 \%) .{ }^{1} \mathrm{H} \mathrm{NMR}\left(\mathrm{C}_{6} \mathrm{D}_{6}, 400\right.$ MHz) $\delta-14.02($ sextet, $J=24 \mathrm{~Hz}, 1 \mathrm{H}), 1.11(\mathrm{~s}, 12 \mathrm{H}), 1.2(\mathrm{br}, 4 \mathrm{H}), 1.29(\mathrm{~s}, 12 \mathrm{H}), 1.5(\mathrm{br}, 4 \mathrm{H})$, $1.70(\mathrm{~d}, J=5 \mathrm{~Hz}, 3 \mathrm{H}) 7.00(\mathrm{t}, J=7 \mathrm{~Hz}, 1 \mathrm{H}), 7.1(\mathrm{br}, 2 \mathrm{H}), 7.59(\mathrm{t}, J=6 \mathrm{~Hz}, 2 \mathrm{H}) \mathrm{ppm} ;{ }^{13} \mathrm{C}\left\{{ }^{1} \mathrm{H}\right\}$ NMR (CDCl $3,100 \mathrm{MHz}) \delta 16.2(\mathrm{~m}), 27.5$ (br), 31.4 (q, J = $13 \mathrm{~Hz}), 96.6(\mathrm{~m}), 121.3$ (s), 126.8 (d, $J=6 \mathrm{~Hz}), 131.9,132 . \mathrm{ppm} ;{ }^{31} \mathrm{P}\left\{{ }^{1} \mathrm{H}\right\} \mathrm{NMR}\left(\mathrm{C}_{6} \mathrm{D}_{6}, 162 \mathrm{MHz}\right) \delta-58.3,43.5 \mathrm{ppm}$; IR (ATR) 1277, 1421, 1475, 1571, $1796 \mathrm{~cm}^{-1}$. Anal. Calcd. C, 43.43; H, 7.86. Found C, 43.81; H, 7.90.

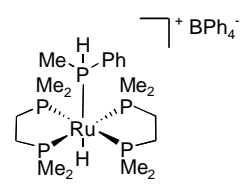

[trans-(dmpe) $\left.)_{2} \mathbf{R u}(\mathbf{H}) \mathbf{P}(\mathbf{H}) \mathrm{MePh}\right]\left[\mathrm{BPh}_{4}\right]\left(\mathbf{2}-\mathbf{B P h}_{4}\right)$. In a nitrogen glove box a 20 $\mathrm{mL}$ scintillation vial was charged with $(\mathrm{dmpe})_{2} \mathrm{Ru}(\mathrm{H}) \mathrm{Cl}(0.100 \mathrm{~g}, 0.23 \mathrm{mmol}, 1.0$ equiv), THF (5 $\mathrm{mL})$ and a stirbar. To this solution sodium tetraphenylborate $(0.080 \mathrm{~g}, 0.23 \mathrm{mmol}, 1.0$ equiv) and methylphenylphosphine $(0.030 \mathrm{~mL}, 0.23 \mathrm{mmol}, 1.0$ equiv) were added via pipette. The solution was stirred overnight, and then filtered through a glass plug and concentrated to afford the desired product as a white solid $(0.120 \mathrm{~g}, 60 \%) .{ }^{1} \mathrm{H}$ NMR (THF- $\left.d_{8}, 400 \mathrm{MHz}\right) \delta-11.85$ (dq, $J=70,24 \mathrm{~Hz}, 1 \mathrm{H}), 1.36(\mathrm{~s}, 12 \mathrm{H}), 1.56(\mathrm{~s}, 12 \mathrm{H}), 1.58(\mathrm{t}, J=6 \mathrm{~Hz}, 3 \mathrm{H}), 1.7(\mathrm{br}, 8 \mathrm{H}), 5.41(\mathrm{dm}, J$ $=312 \mathrm{~Hz}, 1 \mathrm{H}), 6.70(\mathrm{t}, 7 \mathrm{~Hz}, 4 \mathrm{H}), 6.85(\mathrm{t}, J=8 \mathrm{~Hz}, 8 \mathrm{H}), 7.28(\mathrm{br}, 8 \mathrm{H}), 7.33$ (br, 5H) ppm; ${ }^{13} \mathrm{C}\left\{{ }^{1} \mathrm{H},{ }^{31} \mathrm{P}\right\}$ NMR (THF- $\left.d_{8}, 125 \mathrm{MHz}\right) \delta 15.8,17.5,18.0,31.7,121.8,125.7,129.9,130.0$, 131.2, 137.3, 140.5, 165.3 (q, ${ }^{13} \mathrm{C}-\mathrm{B}$ coupling, $\left.J=50 \mathrm{~Hz}\right) \mathrm{ppm} ;{ }^{31} \mathrm{P}\left\{{ }^{1} \mathrm{H}\right\}$ NMR (THF- $d_{8}, 162$ 
MHz) $\delta-16.5$ (q, $J=25 \mathrm{~Hz}$ ), 39.1 (br) ppm; IR (Nujol) 925, 1425, $1860 \mathrm{~cm}^{-1}$. Anal. Calcd. C, 61.86; H, 7.61. Found C, 62.21; H, 7.43.

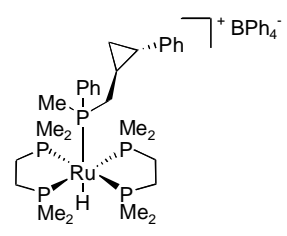

[trans-(dmpe) ${ }_{2} \mathbf{R u}(\mathrm{H}) \mathrm{P}($ methylcyclopropylbenzene)MePh][Br] (5-Br). In a nitrogen glove box a $20 \mathrm{~mL}$ scintillation vial was charged with 1 ( $0.105 \mathrm{~g}, 0.2 \mathrm{mmol}, 1.0$ equiv), THF (2 mL) and a stirbar. A solution of 4 (42 mg, $0.2 \mathrm{mmol}, 1.0$ equiv) in THF (2 mL) was added via pipette. The mixture was stirred for $4 \mathrm{~h}$. The resulting suspension was filtered through a medium frit; the white solid collected was a mixture of the desired product contaminated with trace amounts of $(\mathrm{dmpe})_{2} \mathrm{Ru}(\mathrm{H}) \mathrm{Br}$. Recrystallization from a concentrated solution in THF cooled to $-30{ }^{\circ} \mathrm{C}$ afforded a 1:1 mixture of product diastereomers as a white crystalline solid $(0.098 \mathrm{~g}, 66 \%) .{ }^{1} \mathrm{H}$ NMR $\left(\mathrm{THF}-d_{8}, 500 \mathrm{MHz}\right) \delta-12.7$ ( ddq $, J=71,25,11 \mathrm{~Hz}$, 2H), $0.80(\mathrm{br}, 4 \mathrm{H}), 0.90(\mathrm{br}, 4 \mathrm{H}), 1.34(\mathrm{~s}, 24 \mathrm{H}), 1.52$ (s, 24H), 1.54 (br, 16H), 1.69 (d, J = $5 \mathrm{~Hz}$ 3H), $1.79(\mathrm{~d}, 3 \mathrm{H}, J=5 \mathrm{~Hz}, 3 \mathrm{H}), 2.71(\mathrm{br} \mathrm{d}, J=16 \mathrm{~Hz}, 2 \mathrm{H}), 2.79(\mathrm{br} \mathrm{d}, J=16 \mathrm{~Hz}, 2 \mathrm{H}), 6.69$ (d, 7 $\mathrm{Hz}, 2 \mathrm{H}), 6.84(\mathrm{~d}, 8 \mathrm{~Hz}, 2 \mathrm{H}), 7.00-7.15(\mathrm{~m}, 6 \mathrm{H}), 7.35-7.55(\mathrm{~m}, 10 \mathrm{H}) \mathrm{ppm} ;{ }^{13} \mathrm{C}\left\{{ }^{1} \mathrm{H},{ }^{31} \mathrm{P}\right\} \mathrm{NMR}$ $\left(\mathrm{THF}-d_{8}, 125 \mathrm{MHz}\right) \delta 15.3,17.1,17.3,17.5,17.6,18.4,19.2,22.3,24.9,26.6,30.7,31.0,39.1$, $39.2,124.9,125.0,125.1,127.8,128.0,128.3,128.8,129.5,129.6 \mathrm{ppm} ;{ }^{31} \mathrm{P}\left\{{ }^{1} \mathrm{H}\right\} \mathrm{NMR}\left(\mathrm{THF}-d_{8}\right.$, $162 \mathrm{MHz}) \delta 4.3(\mathrm{q}, J=24 \mathrm{~Hz}), 5.9(\mathrm{q}, J=24 \mathrm{~Hz}), 39.7(\mathrm{~m}) \mathrm{ppm}$; IR (ATR) 698, 890, 1065, 1427, $1871 \mathrm{~cm}^{-1}$. Anal. Calcd. C, 47.29; H, 7.12. Found C, 47.50; H, 7.39.

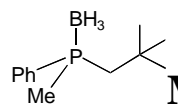

Methyl-(2,2-dimethylpropyl)phenylphosphine borane (6). Note that this phosphine borane was not prepared by the general procedure described above. In a nitrogen glovebox a 50 $\mathrm{mL}$ glass-walled vessel equipped with a vacuum stopcock was charged with $1(0.105 \mathrm{~g}, 0.2$ mmol, 1.0 equiv), 2,2-dimethyl-1-bromopropane ( $0.025 \mathrm{~mL}, 0.2 \mathrm{mmol}, 1.0$ equiv), THF (10 mL) and a stir bar. The flask was sealed and removed from the glovebox. After heating at $45{ }^{\circ} \mathrm{C}$ for 
$24 \mathrm{~h}$, the solution was cooled to room temperature and a borane solution ( $2 \mathrm{~mL}, 1.0 \mathrm{M}$ in THF) was added via syringe. The mixture was stirred for $1 \mathrm{~h}$, and then washed with $\mathrm{NH}_{4} \mathrm{Cl}$ (sat. aq.), dried with $\mathrm{MgSO}_{4}$, filtered, and concentrated under reduced pressure to afford the desired product as a white solid $(0.034 \mathrm{~g}, 82 \%) .{ }^{1} \mathrm{H} \mathrm{NMR}\left(\mathrm{CDCl}_{3}, 400 \mathrm{MHz}\right) \delta$ 0.5-1.2 (br, $\left.\mathrm{BH}, 3 \mathrm{H}\right)$, $0.97(\mathrm{~s}, 9 \mathrm{H}), 1.51(\mathrm{~d}, J=10 \mathrm{~Hz}, 3 \mathrm{H}), 1.87(\mathrm{dd}, J=15,10 \mathrm{~Hz}, 1 \mathrm{H}), 2.02(\mathrm{t}, J=14 \mathrm{~Hz}, 1 \mathrm{H}), 7.46$ $(\mathrm{m}, 3 \mathrm{H}), 7.76(\mathrm{~m}, 2 \mathrm{H}) \mathrm{ppm} ;{ }^{13} \mathrm{C}\left\{{ }^{1} \mathrm{H}\right\} \mathrm{NMR}\left(\mathrm{CDCl}_{3}, 100 \mathrm{MHz}\right) \delta 14.3(\mathrm{~d}, J=41 \mathrm{~Hz}), 31.3(\mathrm{~d}, J=$ $6 \mathrm{~Hz}), 32.2(\mathrm{~s}), 42.7$ (d, $J=31 \mathrm{~Hz}), 128.7$ (d, $J=10 \mathrm{~Hz}), 131.0(\mathrm{~s}), 131.4(\mathrm{~d}, J=9 \mathrm{~Hz}), 132.0(\mathrm{~s})$ ppm; ${ }^{31} \mathrm{P}\left\{{ }^{1} \mathrm{H}\right\} \mathrm{NMR}\left(\mathrm{CDCl}_{3}, 162 \mathrm{MHz}\right) \delta 4.1$ (m) ppm; IR (ATR) 693, 744, 897, 1064, 1437, 2369, $2957 \mathrm{~cm}^{-1}$. HR FAB-MS (M-BH${ }^{+}$) Cacld: 194.1224. Found: 194.1224

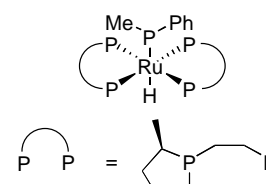

trans-((R,R)-Me-BPE $)_{2} \mathbf{R u}(\mathbf{H}) \mathbf{P M e P h}(\mathbf{7})$. In a nitrogen glove box a $20 \mathrm{~mL}$ scintillation vial was charged with 8-BPh $(0.100 \mathrm{~g}, 0.11 \mathrm{mmol}, 1.0$ equiv), THF (4 mL) and a stirbar. Methylphenylphosphine $(0.014 \mathrm{~mL}, 0.11 \mathrm{mmol}, 1.0$ equiv) was added via syringe. The slurry was stirred for 5 min or until all of the ruthenium complex had dissolved. KHMDS (0.022 $\mathrm{g}, 0.11 \mathrm{mmol}, 1.0$ equiv) in THF (1 mL) was then added. The slurry was stirred for $10 \mathrm{~min}$ and then filtered through a glass plug, and the resulting clear, orange solution was concentrated to afford a yellow solid (0.081 g, $100 \%) .{ }^{1} \mathrm{H}$ NMR (THF- $\left.d_{8}, 400 \mathrm{MHz}\right) \delta-14.29$ ( sextet, 1H, 27 $\mathrm{Hz}), 1.05$ ( quintet, $J=7 \mathrm{~Hz}, 16 \mathrm{H}), 1.15(\mathrm{q}, J=7 \mathrm{~Hz}, 8 \mathrm{H}), 1.32(\mathrm{~d}, J=5 \mathrm{~Hz}, 3 \mathrm{H}), 1.38(\mathrm{q}, J=7$ $\mathrm{Hz}, 8 \mathrm{H}), 1.45-1.53(\mathrm{~m}, 8 \mathrm{H}), 1.56-1.70(\mathrm{~m}, 8 \mathrm{H}), 1.9-2.0(\mathrm{~m}, 8 \mathrm{H}) 6.45(\mathrm{t}, J=7 \mathrm{~Hz}, 1 \mathrm{H}), 6.74(\mathrm{t}, J$ $=7 \mathrm{~Hz}, 2 \mathrm{H}), 7.14(\mathrm{t}, J=7 \mathrm{~Hz}, 1 \mathrm{H}), 7.32(\mathrm{br}, 1 \mathrm{H}) \mathrm{ppm} ;{ }^{13} \mathrm{C}\left\{{ }^{1} \mathrm{H}\right\} \mathrm{NMR}\left(\mathrm{THF}-d_{8}, 125 \mathrm{MHz}\right) \delta$ $14.4,14.8,15.8,15.9,16.0,16.5,16.9,17.0,18.2,18.3,18.4,18.5,19.3,20.6-20.7$ (m), 20.8, 23.0, 23.3, 24.9, 33.5, 33.8, 34.0, 34.5, 34.7, 35.1, 36.4, 36.5, 36.7, 36.8, 37.6, 37.9, 38.2, 40.0$40.1(\mathrm{~m}), 41.1(\mathrm{~m}), 42.4(\mathrm{~m}), 126.6(\mathrm{~m}), 128.9(\mathrm{~m}), 129.7(\mathrm{~m}), 131.9(\mathrm{~m}) \mathrm{ppm} ;{ }^{31} \mathrm{P}\left\{{ }^{1} \mathrm{H}\right\} \mathrm{NMR}$ 
$\left(\mathrm{THF}-d_{8}, 162 \mathrm{MHz}\right) \delta-106.2,76.2,86.4 \mathrm{ppm}$; IR (Nujol) $1861 \mathrm{~cm}^{-1}$. HR FAB-MS $\left(\mathrm{M}+\mathrm{H}^{+}\right)$ Cacld: 743.2896. Found: 743.2871.

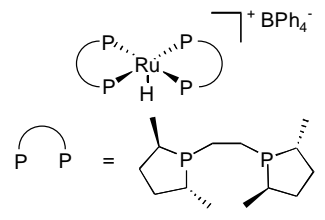

[trans-(1,2-bis $\left.((2 R, 5 R)-2,5 \text {-dimethylphospholano)ethane })_{2} \mathrm{Ru}(\mathrm{H})\right]\left[\mathrm{BPh}_{4}\right]$

$\left(\mathbf{8}-\mathbf{B P h}_{4}\right)$. In a nitrogen glovebox a $20 \mathrm{~mL}$ scintillation vial was charged with $\left[\mathrm{Ru}(\mathrm{H})\left(\mathrm{NH}_{2} \mathrm{NMe}_{2}\right)_{2}(\mathrm{cod})\right]\left[\mathrm{BPh}_{4}\right](0.467 \mathrm{~g}, 0.63 \mathrm{mmol}, 1.0$ equiv), THF (5 mL) and a stirbar. To this solution 1,2-bis((2R,5R)-2,5-dimethylphospholano)ethane (0.326 mg, $1.26 \mathrm{mmol}, 2.0$ equiv) was added via pipette. The solution was stirred overnight and then concentrated to afford a red solid. Recrystallization from a concentrated THF solution afforded the desired product as red needles $(0.525 \mathrm{~g}, 89 \%) .{ }^{1} \mathrm{H}$ NMR (THF- $\left.d_{8}, 400 \mathrm{MHz}\right) \delta-15.0(\mathrm{br}, 1 \mathrm{H}), 1.0-2.2(\mathrm{br} \mathrm{m}, 56 \mathrm{H}), 6.71$ $(\mathrm{t}, J=7 \mathrm{~Hz}, 4 \mathrm{H}), 6.85(\mathrm{t}, J=7 \mathrm{~Hz}, 8 \mathrm{H}), 7.27(\mathrm{br}, 8 \mathrm{H}) \mathrm{ppm} ;{ }^{13} \mathrm{C}\left\{{ }^{1} \mathrm{H},{ }^{31} \mathrm{P}\right\} \mathrm{NMR}\left(\mathrm{THF}-d_{8}, 125\right.$ MHz) $\delta 11.4,11.9,33.9,34.7,35.0,38.5,118.7,119.9,122.5,134.2,162.0\left(\mathrm{q},{ }^{13} \mathrm{C}-\mathrm{B}\right.$ coupling, $J$ $=49 \mathrm{~Hz})$ ppm; ${ }^{31} \mathrm{P}\left\{{ }^{1} \mathrm{H}\right\} \mathrm{NMR}\left(\mathrm{THF}-d_{8}, 162 \mathrm{MHz}\right) \delta 69.6$ (br), 72.2 (br) ppm; IR (Nujol) 2174 $\mathrm{cm}^{-1}$. Anal. Calcd. C, 64.66; H, 8.03. Found C, 64.82; H, 8.03. ${ }^{4}$<smiles>[B][PH](Cc1ccccc1)(c1ccccc1)c1ccccc1</smiles>

Benzylmethylphenylphosphine borane (9). Flash chromatography (80:20 hexanes: $\left.\mathrm{CH}_{2} \mathrm{Cl}_{2}\right)$ furnished product as a yellow solid $(42 \mathrm{mg}, 91 \%) .{ }^{1} \mathrm{H} \mathrm{NMR}\left(\mathrm{CDCl}_{3}\right.$, $400 \mathrm{MHz}) \delta 7.63-7.41(\mathrm{~m}, 5 \mathrm{H}), 7.21(\mathrm{~m}, 3 \mathrm{H}), 6.92(\mathrm{~m}, 2 \mathrm{H}) 3.21(\mathrm{~d}, J=8.4 \mathrm{~Hz}, 2 \mathrm{H}), 1.51(\mathrm{~d}, J=$ $10 \mathrm{~Hz}, 3 \mathrm{H}), 0.69$ (br q, 3H). ${ }^{13} \mathrm{C}\left\{{ }^{1} \mathrm{H}\right\} \mathrm{NMR}\left(\mathrm{CDCl}_{3}, 100 \mathrm{MHz}\right) \delta 132.3(\mathrm{~d}, J=7 \mathrm{~Hz}), 131.7(\mathrm{~d}, 9$ $\mathrm{Hz}), 131.4,129.7(\mathrm{~d}, J=4 \mathrm{~Hz}), 129.0,128.5(\mathrm{~d}, J=10 \mathrm{~Hz}), 128.2(\mathrm{~d}, J=2 \mathrm{~Hz}), 126.9(\mathrm{~d}, J=3$ $\mathrm{Hz}), 35.8(\mathrm{~d}, J=31 \mathrm{~Hz}), 8.8(\mathrm{~d}, J=39 \mathrm{~Hz}) .{ }^{31} \mathrm{P}\left\{{ }^{1} \mathrm{H}\right\} \mathrm{NMR}\left(\mathrm{CDCl}_{3}, 162 \mathrm{MHz}\right) \delta 10.3\left(\mathrm{q}, J_{\mathrm{B}-\mathrm{P}}=\right.$ 63 Hz). IR: 3058, 3029, 2917, 2368, 2342, 2258, 1601, 1495, 1436, 1416, 1138, 1063, 915, 897, 845, 791, 744, $691 \mathrm{~cm}^{-1}$. HRMS $\left(\mathrm{FAB}^{+}\right)$calcd for $\left[\mathrm{C}_{14} \mathrm{H}_{15} \mathrm{BP}\right]^{+}$225.1004, Found 225.1005. Analysis of enantiomers by chiral HPLC (Chiralcel OJ Column, flow rate $0.9 \mathrm{~mL} / \mathrm{min}, 94: 6$ 
hexanes:i-PrOH, $\mathrm{T}_{\mathrm{r}}$ minor 33.44, major $37.58 \mathrm{~min}$ ) determined the ee to be $75 \%$. Recrystallization of 7 from ethanol at $-30{ }^{\circ} \mathrm{C}$ afforded the product as a white crystalline powder (12 mg, 29\%) in $92 \%$ ee.

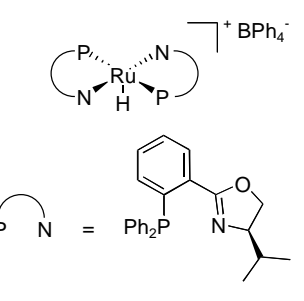

$\left[(\boldsymbol{R})-(\boldsymbol{i}-\mathbf{P r}-\mathbf{P H O X})_{2} \mathbf{R u}(\mathbf{H})\right]\left[\mathrm{BPh}_{4}\right](\mathbf{1 0}) . \quad$ To a 50-mL glass-walled vessel equipped with a vacuum stopcock was added $\left(\mathrm{PPh}_{3}\right)_{3} \mathrm{Ru}(\mathrm{H})(\mathrm{Cl})(600 \mathrm{mg}, 0.65 \mathrm{mmol}, 1.0$ equiv), and $(R)$ - $i$-Pr-PHOX (485 mg, $1.3 \mathrm{mmol}, 2.0$ equiv), and THF (10 mL). The sealed vessel was brought out of the glovebox and then degassed by three freeze-pump-thaw cycles. The solution was then heated to $105{ }^{\circ} \mathrm{C}$; after $18 \mathrm{~h}$, the solution was cooled to room temperature and concentrated to $5 \mathrm{~mL}$. Under a nitrogen atmosphere, $\mathrm{NaBPh}_{4}(222 \mathrm{mg}, 0.65 \mathrm{mmol}, 1.0$ equiv) was added as a 1.0 M THF solution. After $10 \mathrm{~h}$ at room temperature, the reaction mixture was filtered through a pad of Celite. THF was removed in vacuo to afford a dark orange-red solid. The solid was then washed with $\mathrm{Et}_{2} \mathrm{O}(3 \times 1 \mathrm{~mL})$ and benzene $(3 \times 1 \mathrm{~mL})$. Further purification was accomplished by precipitation of $\mathbf{1 0}$ from a saturated THF solution by slow vapor diffusion of $\mathrm{Et}_{2} \mathrm{O}$ at room temperature. $\mathbf{1 0}$ was obtained as a red crystalline solid (564 $\mathrm{mg}, 74 \%$ ): $\mathrm{mp}$ 209.5-211 ${ }^{\circ} \mathrm{C}(\mathrm{dec})$. Note that we cannot yet unambiguously assign the stereochemistry of this complex in solution. ${ }^{1} \mathrm{H}$ NMR $\left(\mathrm{THF}-d_{8}, 400 \mathrm{MHz}\right) \delta 8.08(\mathrm{~m}, 1 \mathrm{H}), 8.01(\mathrm{~m} 1 \mathrm{H}), 7.90-7.81(\mathrm{~m}$ 3H), 7.64-7.14 (m, 22H), $6.86(\mathrm{~m}, 12 \mathrm{H}), 6.68(\mathrm{~m}, 8 \mathrm{H}), 5.98(\mathrm{~m}, 1 \mathrm{H}), 4.49(\mathrm{~m}, 3 \mathrm{H}), 4.27$ (br t, 1H), $4.22(\mathrm{~m}, 1 \mathrm{H}), 3.98$ (br d, 1H), $2.80(\mathrm{~m}, 1 \mathrm{H}), 0.82$ (d, J = 7.2 Hz, 3H), 0.55 (br s, 4H), 0.33 $(\mathrm{d}, J=6.8 \mathrm{~Hz}, 3 \mathrm{H}),-0.21(\mathrm{br} \mathrm{s}, 3 \mathrm{H}),-20.9(\mathrm{Ru}-H, \mathrm{dd}, J=27.6,27.2,35.8 \mathrm{~Hz}, 1 \mathrm{H}) .{ }^{11} \mathrm{~B}\left\{{ }^{1} \mathrm{H}\right\}$ NMR (THF- $\left.\left.d_{8}\right), 128 \mathrm{MHz}\right) \delta-8.5 .{ }^{31} \mathrm{P}\left\{{ }^{1} \mathrm{H}\right\} \mathrm{NMR}\left(\mathrm{THF}-d_{8}, 162 \mathrm{MHz}\right) \delta 67.4(\mathrm{~d}, J=26 \mathrm{~Hz})$, $59.9(\mathrm{~d}, J=24 \mathrm{~Hz}) . \quad$ IR: 3054, 3000, 2960, 2931, 2361, 2342, 2048, 1644, 1627, 1580, 1479, 1434, 1372, 1238, 1142, 1094, 1058, 998, 955, 743, $694 \mathrm{~cm}^{-1}$. Anal. Calcd for $\mathrm{C}_{72} \mathrm{H}_{69} \mathrm{BN}_{2} \mathrm{O}_{2} \mathrm{P}_{2} \mathrm{Ru}: \mathrm{C}$ 74.03, H 5.95, N 2.40; Found: C 73.65, H 5.98, N 2.39. 


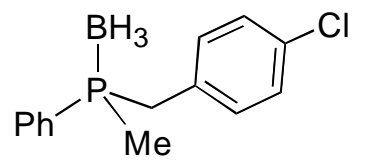

$\alpha-4$-(chlorotolyl)methylphenylphosphine borane. Flash

chromatography (80:20 hexanes: $\mathrm{CH}_{2} \mathrm{Cl}_{2}$ ) furnished product as a yellow oil, which solidified upon standing $(51 \mathrm{mg}, 96 \%) .{ }^{1} \mathrm{H} \mathrm{NMR}\left(\mathrm{CDCl}_{3}, 400 \mathrm{MHz}\right) \delta 7.58-7.42(\mathrm{~m}, 5 \mathrm{H}), 7.18(\mathrm{~d}, J=8$ $\mathrm{Hz}, 2 \mathrm{H}), 6.84(\mathrm{~d}, J=6.8 \mathrm{~Hz}, 2 \mathrm{H}), 3.17(\mathrm{~d}, J=10.8 \mathrm{~Hz}, 2 \mathrm{H}), 1.53(\mathrm{~d}, J=9.6 \mathrm{~Hz}, 3 \mathrm{H}), 0.77$ (br q, 3H). ${ }^{13} \mathrm{C}\left\{{ }^{1} \mathrm{H}\right\} \operatorname{NMR}\left(\mathrm{CDCl}_{3}, 100 \mathrm{MHz}\right) \delta 132.9(\mathrm{~d}, J=3 \mathrm{~Hz}), 131.6(\mathrm{~d}, J=9 \mathrm{~Hz}), 131.5(\mathrm{~d}, J=$ $2 \mathrm{~Hz}), 130.9(\mathrm{~d}, J=4 \mathrm{~Hz}), 130.7(\mathrm{~d}, 7 \mathrm{~Hz}), 128.7(\mathrm{~d}, J=10 \mathrm{~Hz}), 128.3(\mathrm{~d}, J=3 \mathrm{~Hz}), 128.0,35.3$ $(\mathrm{d}, J=31 \mathrm{~Hz}), 9.0(\mathrm{~d}, J=38 \mathrm{~Hz}) .{ }^{31} \mathrm{P}\left\{{ }^{1} \mathrm{H}\right\} \mathrm{NMR}\left(\mathrm{CDCl}_{3}, 162 \mathrm{MHz}\right) \delta 10.7\left(\mathrm{q}, J_{\mathrm{B}-\mathrm{P}}=71 \mathrm{~Hz}\right)$. IR: 3057, 2913, 2395, 2375, 2342, 2261, 1490, 1435, 1407, 1091, 1067, 1014, 920, 900, 837, $734,691 \mathrm{~cm}^{-1}$. HRMS $\left(\mathrm{FAB}^{+}\right)$calcd for $\left[\mathrm{C}_{14} \mathrm{H}_{14} \mathrm{BClP}\right]^{+}$259.0615, Found 259.0619. Analysis of enantiomers by chiral HPLC (Chiralpak AS Column, flow rate $1.1 \mathrm{~mL} / \mathrm{min}, 96: 4$ hexanes:iPrOH, $\mathrm{T}_{\mathrm{r}}$ major 11.86 , minor $13.29 \mathrm{~min}$ ) determined the ee to be $41 \%$.<smiles>[B][PH](C)(Cc1ccc(C)cc1)c1ccccc1</smiles>

$\alpha$-(p-xylyl)methylphenylphosphine borane. Flash chromatography (70:30 hexanes: $\mathrm{CH}_{2} \mathrm{Cl}_{2}$ ) furnished product as a yellow oil (39 $\left.\mathrm{mg}, 80 \%\right) .{ }^{1} \mathrm{H} \mathrm{NMR}\left(\mathrm{CDCl}_{3}, 400\right.$ MHz) $\delta 7.60-7.42(\mathrm{~m}, 5 \mathrm{H}), 3.19(\mathrm{~m}, 2 \mathrm{H}), 2.30(\mathrm{~s}, 3 \mathrm{H}), 1.50(\mathrm{~d}, J=10 \mathrm{~Hz}, 3 \mathrm{H}), 0.79$ (br q, 3H). ${ }^{13} \mathrm{C}\left\{{ }^{1} \mathrm{H}\right\} \mathrm{NMR}\left(\mathrm{CDCl}_{3}, 100 \mathrm{MHz}\right) \delta 136.6,131.7(\mathrm{~d}, J=9 \mathrm{~Hz}), 131.3,129.5(\mathrm{~d}, J=4 \mathrm{~Hz}), 129.0$, 128.9, 128.6, 128.5, $35.4(\mathrm{~d}, J=31 \mathrm{~Hz}), 21.0,8.8(\mathrm{~d}, J=39 \mathrm{~Hz}) .{ }^{31} \mathrm{P}\left\{{ }^{1} \mathrm{H}\right\} \mathrm{NMR}\left(\mathrm{CDCl}_{3}, 162\right.$ MHz) $\delta 10.0$ (q, $J_{\text {B-P }}=75$ Hz). IR: 3057, 2921, 2854, 2386, 2369, 2343, 1513, 1436, 1148, $1117,1068,914,898,837,818,785,746,723,690 \mathrm{~cm}^{-1}$. HRMS $\left(\mathrm{FAB}^{+}\right)$calcd for $\left[\mathrm{C}_{15} \mathrm{H}_{17} \mathrm{BP}\right]^{+}$ 239.1161, Found 239.1167. Analysis of enantiomers by chiral HPLC (Chiralpak AS Column, flow rate 1.1 mL/min, 99.5:0.5 hexanes:i-PrOH, $\mathrm{T}_{\mathrm{r}}$ major 24.29, minor 29.28 min) determined the ee to be $83 \%$. 
$\alpha-(p$-methoxy)methylphenylphosphine borane. Flash chromatography (60:40 hexanes: $\left.\mathrm{CH}_{2} \mathrm{Cl}_{2}\right)$ furnished product as a yellow oil $(44 \mathrm{mg}, 85 \%) .{ }^{1} \mathrm{H},{ }^{13} \mathrm{C}\left\{{ }^{1} \mathrm{H}\right\}$ and ${ }^{31} \mathrm{P}\left\{{ }^{1} \mathrm{H}\right\}$ NMR spectra agree with literature values. ${ }^{5}$ Analysis of enantiomers by chiral HPLC (Chiralpak AS Column, flow rate $0.8 \mathrm{~mL} / \mathrm{min}, 98: 2$ hexanes:i-PrOH, $\mathrm{T}_{\mathrm{r}}$ major 26.75 , minor 30.29) determined the ee to be $85 \%$.

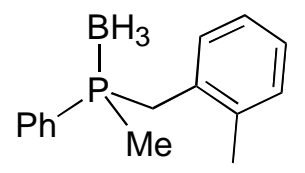

$\alpha$-(o-xylyl)methylphenylphosphine borane. Flash chromatography (80:20 hexanes: $\left.\mathrm{CH}_{2} \mathrm{Cl}_{2}\right)$ furnished product as a yellow solid $(45 \mathrm{mg}, 92 \%) .{ }^{1} \mathrm{H} \mathrm{NMR}\left(\mathrm{CDCl}_{3}\right.$, $400 \mathrm{MHz}) \delta 7.52(\mathrm{~m}, 3 \mathrm{H}), 7.41(\mathrm{~m}, 2 \mathrm{H}), 7.17-7.07(\mathrm{~m}, 3 \mathrm{H}), 6.89(\mathrm{~m}, 1 \mathrm{H}), 3.24(\mathrm{~m}, 2 \mathrm{H}), 1.96(\mathrm{~s}$, $3 \mathrm{H}), 1.56(\mathrm{~d}, J=10 \mathrm{~Hz}, 3 \mathrm{H}), 0.83(\mathrm{brq}, 3 \mathrm{H}) .{ }^{13} \mathrm{C}\left\{{ }^{1} \mathrm{H}\right\} \mathrm{NMR}\left(\mathrm{CDCl}_{3}, 100 \mathrm{MHz}\right) \delta 136.9(\mathrm{~d}, J=$ $4 \mathrm{~Hz}), 131.8(\mathrm{~d}, J=9 \mathrm{~Hz}), 131.4(\mathrm{~d}, J=2 \mathrm{~Hz}), 130.9$ (d, $J=8 \mathrm{~Hz}), 130.6(\mathrm{~d}, J=3 \mathrm{~Hz}), 130.3(\mathrm{~d}$, $J=4 \mathrm{~Hz}), 129.2,128.5(\mathrm{~d}, J=10 \mathrm{~Hz}), 127.1(\mathrm{~d}, J=3 \mathrm{~Hz}), 125.7(\mathrm{~d}, J=2 \mathrm{~Hz}), 32.5(\mathrm{~d}, J=30$ $\mathrm{Hz}), 19.7,9.1(\mathrm{~d}, J=39 \mathrm{~Hz}) .{ }^{31} \mathrm{P}\left\{{ }^{1} \mathrm{H}\right\} \mathrm{NMR}\left(\mathrm{CDCl}_{3}, 162 \mathrm{MHz}\right) \delta 10.4\left(\mathrm{q}, J_{\mathrm{B}-\mathrm{P}}=71 \mathrm{~Hz}\right) . \quad \mathrm{IR}$ : 3057, 3025, 2904, 2373, 2342, 2250, 1494, 1438, 1417, 1116, 1063, 901, 823, 786, 738, 692 $\mathrm{cm}^{-1}$. HRMS $\left(\mathrm{FAB}^{+}\right)$calcd for $\left[\mathrm{C}_{15} \mathrm{H}_{17} \mathrm{BP}\right]^{+}$239.1161, Found 239.1165. Analysis of enantiomers by chiral HPLC (Chiralcel OJ Column, flow rate $0.9 \mathrm{~mL} / \mathrm{min}, 94: 6$ hexanes:i-PrOH, $\mathrm{T}_{\mathrm{r}}$ major 30.54 , minor $58.41 \mathrm{~min}$ ) determined the ee to be $59 \%$.

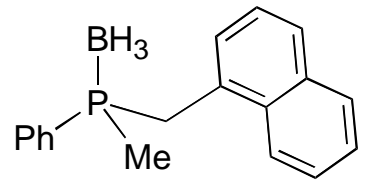

$\alpha-(1-n a p t h a l e n y l m e t h y l) m e t h y l p h e n y l p h o s p h i n e \quad$ borane. Flash chromatography (80:20 hexanes: $\mathrm{CH}_{2} \mathrm{Cl}_{2}$ ) furnished product as a yellow oil (54 mg, 96\%). ${ }^{1} \mathrm{H}$ NMR $\left(\mathrm{CDCl}_{3}, 400 \mathrm{MHz}\right) \delta 7.82(\mathrm{~d}, J=8 \mathrm{~Hz}, 1 \mathrm{H}), 7.75(\mathrm{~d}, J=8 \mathrm{~Hz}, 1 \mathrm{H}), 7.65(\mathrm{~d}, J=8.4 \mathrm{~Hz}$, 1H), 7.58-7.31 (m, 8H), $7.06(\mathrm{~d}, J=6.4 \mathrm{~Hz}, 1 \mathrm{H}), 3.70(\mathrm{~d}, J=10.4 \mathrm{~Hz}, 2 \mathrm{H}), 1.46(\mathrm{~d}, J=9.6 \mathrm{~Hz}$, $3 \mathrm{H}), 0.89$ (br q, 3H). ${ }^{13} \mathrm{C}\left\{{ }^{1} \mathrm{H}\right\} \mathrm{NMR}\left(\mathrm{CDCl}_{3}, 100 \mathrm{MHz}\right) \delta 133.7,132.7(\mathrm{~d}, J=9 \mathrm{~Hz}), 131.9$, 
$131.7(\mathrm{~d}, J=9 \mathrm{~Hz}), 131.3,129.2,128.9(\mathrm{~d}, J=9 \mathrm{~Hz}), 128.6,128.5(\mathrm{~d}, J=10 \mathrm{~Hz}), 128.2(\mathrm{~d}, J=5$ $\mathrm{Hz}), 127.8(\mathrm{~d}, J=3 \mathrm{~Hz}), 125.9,125.7,125.0(\mathrm{~d}, J=3 \mathrm{~Hz}), 123.8,32.2(\mathrm{~d}, J=31 \mathrm{~Hz}), 9.0(\mathrm{~d}, J=$ $38 \mathrm{~Hz}) .{ }^{31} \mathrm{P}\left\{{ }^{1} \mathrm{H}\right\} \mathrm{NMR}\left(\mathrm{CDCl}_{3}, 162 \mathrm{MHz}\right) \delta 11.4\left(\mathrm{q}, J_{\mathrm{B}-\mathrm{P}}=58 \mathrm{~Hz}\right) . \quad$ IR: 3056, 2918, 2372, 2336, 2252, 1595, 1510, 1435, 1417, 1114, 1061, 1014, 899, 802, 781, 739, $692 \mathrm{~cm}^{-1}$. HRMS $\left(\mathrm{EI}^{+}\right)$ calcd for $\left[\mathrm{C}_{18} \mathrm{H}_{19} \mathrm{BP}\right]^{+}$277.1317, Found 277.1356. Analysis of enantiomers by chiral HPLC (Chiralcel OJ Column, flow rate $1.1 \mathrm{~mL} / \mathrm{min}, 85: 15$ hexanes:i-PrOH, $\mathrm{T}_{\mathrm{r}}$ major 31.04, minor $68.20 \mathrm{~min}$ ) determined the ee to be $59 \%$.

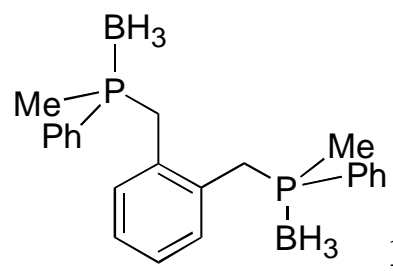

\section{1,2-Bis((methylphenylphosphinoborane)methyl)benzene.}

Flash

chromatography (70:30 hexanes:EtOAc) furnished product as a white solid (33 mg, 87\%); isolated as a mixture of $C_{2}$ and meso diastereomers. ${ }^{1} \mathrm{H} \mathrm{NMR}\left(\mathrm{CDCl}_{3}, 400 \mathrm{MHz}\right) \delta 7.55-7.43$ (m, 6H), 7.42-7.38 (m, 4H), $7.08(\mathrm{~m}, 2 \mathrm{H}), 6.80(\mathrm{~m}, 2 \mathrm{H}), 3.01(\mathrm{~m}, 4 \mathrm{H}), 1.50(\mathrm{~m}, 6 \mathrm{H}), 1.02-0.31$ (m, 6H). ${ }^{13} \mathrm{C}\left\{{ }^{1} \mathrm{H}\right\} \mathrm{NMR}\left(\mathrm{CDCl}_{3}, 100 \mathrm{MHz}\right) \delta 131.8-131.6(\mathrm{~m}), 131.2(\mathrm{~m}), 128.8(\mathrm{~m}), 128.7(\mathrm{~m})$, $127.1(\mathrm{~m}), 32.9(\mathrm{~d}, J=8 \mathrm{~Hz}), 32.6(\mathrm{~d}, J=9 \mathrm{~Hz}), 10.0(\mathrm{~d}, J=11 \mathrm{~Hz}), 9.6(\mathrm{~d}, J=10 \mathrm{~Hz}) .{ }^{31} \mathrm{P}\left\{{ }^{1} \mathrm{H}\right\}$ NMR (CDCl $3,162 \mathrm{MHz}) \delta 10.4$ (m). IR: 3057, 2962, 2917, 2852, 2376, 2356, 2264, 1488, 1437, 1416, 1117, 1061, 922, 901, 785, 761, 743, $691 \mathrm{~cm}^{-1}$. HRMS $\left(\mathrm{FAB}^{+}\right)$calcd for $\left[\mathrm{C}_{22} \mathrm{H}_{25} \mathrm{~B}_{2} \mathrm{P}_{2}\right]^{+}$373.1618, Found 373.1619. Analysis of enantiomers by chiral HPLC (Chiralpak AD Column, flow rate $1.0 \mathrm{~mL} / \mathrm{min}, 80: 20$ hexanes:i-PrOH, $C_{2}$ : $\mathrm{T}_{\mathrm{r}}$ minor 8.06 , major $12.02 \mathrm{~min}$, meso: $\mathrm{T}_{\mathrm{r}} 9.05 \mathrm{~min}$ ) determined the ee to be $74 \%$. Ratio of $C_{2}$ :meso diastereomers found to be 33:67. 


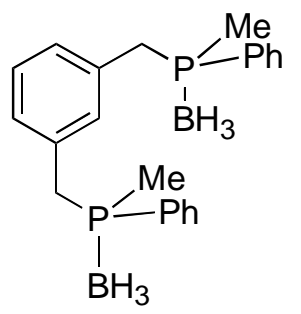

1,3-Bis((methylphenylphosphinoborane)methyl)benzene.

Flash

chromatography (75:25 hexanes:EtOAc) furnished product as a yellow oil (33 mg, 86\%); isolated as a mixture of $C_{2}$ and meso diastereomers. ${ }^{1} \mathrm{H}$ NMR $\left(\mathrm{CDCl}_{3}, 400 \mathrm{MHz}\right) \delta 7.58-7.42$ (m, 10H), $7.02(\mathrm{t} J=7.6 \mathrm{~Hz}, 1 \mathrm{H}), 6.76-6.63(\mathrm{~m}, 3 \mathrm{H}), 3.12(\mathrm{~m}, 4 \mathrm{H}), 1.52-1.44(\mathrm{~m}, 6 \mathrm{H}), 1.08-$ $0.42(\mathrm{~m}, 6 \mathrm{H}) .{ }^{13} \mathrm{C}\left\{{ }^{1} \mathrm{H}\right\} \operatorname{NMR}\left(\mathrm{CDCl}_{3}, 100 \mathrm{MHz}\right) \delta 132.7(\mathrm{~m}), 131.7(\mathrm{~m}), 131.4(\mathrm{~m}), 128.6(\mathrm{~m})$, $128.38(\mathrm{~m}), 35.7(\mathrm{~d}, J=32 \mathrm{~Hz}), 9.3(\mathrm{~d}, J=38 \mathrm{~Hz}) .{ }^{31} \mathrm{P}\left\{{ }^{1} \mathrm{H}\right\} \mathrm{NMR}\left(\mathrm{CDCl}_{3}, 162 \mathrm{MHz}\right) \delta 10.8$ (m). IR: 3056, 2961, 2918, 2853, 2371, 2337, 2253, 1487, 1436, 1415, 1134, 1115, 1061, 942 , 900, 806, 739, $691 \mathrm{~cm}^{-1}$. HRMS $\left(\mathrm{FAB}^{+}\right)$calcd for $\left[\mathrm{C}_{22} \mathrm{H}_{25} \mathrm{~B}_{2} \mathrm{P}_{2}\right]^{+} 373.1618$, Found 373.1622. Analysis of enantiomers by chiral HPLC (Chiralpak AS Column, flow rate $1.1 \mathrm{~mL} / \mathrm{min}, 95: 5$ hexanes:EtOH, $C_{2}$ : $\mathrm{T}_{\mathrm{r}}$ major 21.59 , minor $30.35 \mathrm{~min}$, meso: $\mathrm{T}_{\mathrm{r}} 26.76 \mathrm{~min}$ ) determined the ee to be $95 \%$. Ratio of $C_{2}$ :meso diastereomers found to be $74: 26$.

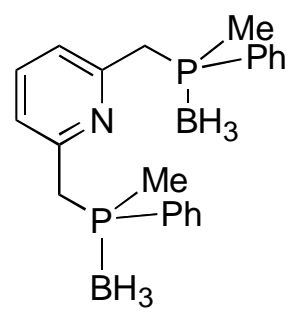

\section{1,3-Bis((methylphenylphosphinoborane)methyl)pyridine.}

Flash

chromatography (60:40 hexanes:EtOAc) furnished product as a yellow oil $(34 \mathrm{mg}, 89 \%) .{ }^{1} \mathrm{H}$ NMR $\left(\mathrm{CDCl}_{3}, 400 \mathrm{MHz}\right) \delta 7.68-7.59(\mathrm{~m}, 4 \mathrm{H}), 7.54-7.40(\mathrm{~m}, 7 \mathrm{H}), 6.87-6.81(\mathrm{~m}, 2 \mathrm{H}), 3.38-3.34$ (m, 4H), 1.62-1.49 (m, 6H), $0.78($ br q, $6 \mathrm{H}) .{ }^{13} \mathrm{C}\left\{{ }^{1} \mathrm{H}\right\}$ NMR $\left(\mathrm{CDCl}_{3}, 100 \mathrm{MHz}\right) \delta 153.5(\mathrm{~m})$, 136.6, 131.7 (dd, $J=2,9 \mathrm{~Hz}), 131.5,129.3(\mathrm{~d}, J=10 \mathrm{~Hz}), 128.7(\mathrm{~m}), 122.7(\mathrm{~m}), 37.9(\mathrm{~d}, J=11$ $\mathrm{Hz}), 37.6(\mathrm{~d}, J=10 \mathrm{~Hz}), 10.1(\mathrm{~d}, J=7 \mathrm{~Hz}), 9.7(\mathrm{~d}, J=6 \mathrm{~Hz}) .{ }^{31} \mathrm{P}\left\{{ }^{1} \mathrm{H}\right\} \mathrm{NMR}\left(\mathrm{CDCl}_{3}, 162 \mathrm{MHz}\right)$ $\delta 11.5$ (m). IR: 3057, 2960, 2918, 2370, 2336, 2252, 1589, 1573, 1451, 1435, 1413, 1135, 1115 , 1061, 898, 830, 740, $691 \mathrm{~cm}^{-1}$. HRMS $\left(\mathrm{EI}^{+}\right)$calcd for $\left[\mathrm{C}_{21} \mathrm{H}_{26} \mathrm{BNP}_{2}\right]^{+} 365.1634$, Found 
365.1590. Analysis of enantiomers by chiral HPLC (Chiralpak AS Column, flow rate 1.1 $\mathrm{mL} / \mathrm{min}$, 95:5 hexanes:EtOH, $C_{2}$ : $\mathrm{T}_{\mathrm{r}}$ major 20.17, minor $31.62 \mathrm{~min}$, meso: $\mathrm{T}_{\mathrm{r}} 27.07 \mathrm{~min}$ ) determined the ee to be $84 \%$. Ratio of $C_{2}$ :meso diastereomers found to be $58: 42$.

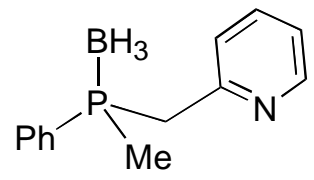

(2-methylpyridyl)methylphenylphosphine

borane.

Flash

chromatography (70:30 hexanes:EtOAc) furnished product as a yellow oil (43 mg, 94\%). ${ }^{1} \mathrm{H}$ NMR $\left(\mathrm{CDCl}_{3}, 400 \mathrm{MHz}\right) \delta 8.48(\mathrm{~d}, J=4.8 \mathrm{~Hz}, 1 \mathrm{H}), 7.64-7.60(\mathrm{~m}, 2 \mathrm{H}), 7.56-7.46(\mathrm{~m} .2 \mathrm{H})$, 7.43-7.39 (m, 2H), $7.14(\mathrm{t}, J=6.4 \mathrm{~Hz}, 1 \mathrm{H}), 7.00(\mathrm{~d}, J=8 \mathrm{~Hz}, 1 \mathrm{H}), 3.41(\mathrm{~m}, 2 \mathrm{H}), 1.61(\mathrm{~d}, J=$ $10.4 \mathrm{~Hz}, 3 \mathrm{H}), 0.60$ (br q, 3H). ${ }^{11} \mathrm{~B}\left\{{ }^{1} \mathrm{H}\right\} \mathrm{NMR}\left(\mathrm{CDCl}_{3}, 128 \mathrm{MHz}\right) \delta-39.1\left(\mathrm{~d}, J_{\mathrm{B}-\mathrm{P}}=58 \mathrm{~Hz}\right)$. ${ }^{13} \mathrm{C}\left\{{ }^{1} \mathrm{H}\right\} \mathrm{NMR}\left(\mathrm{CDCl}_{3}, 100 \mathrm{MHz}\right) \delta 153.75(\mathrm{~d}, J=7 \mathrm{~Hz}), 149.3,136.4,131.7(\mathrm{~d}, J=9 \mathrm{~Hz})$, 131.4, 129.3, $128.7(\mathrm{~d}, J=10 \mathrm{~Hz}), 124.7(\mathrm{~d}, J=4 \mathrm{~Hz}), 122.0,38.0(\mathrm{~d}, J=30 \mathrm{~Hz}), 9.5(\mathrm{~d}, J=38$ Hz). ${ }^{31} \mathrm{P}\left\{{ }^{1} \mathrm{H}\right\} \mathrm{NMR}\left(\mathrm{CDCl}_{3}, 162 \mathrm{MHz}\right) \delta 11.5$ (q, $\left.J_{\mathrm{B}-\mathrm{P}}=65 \mathrm{~Hz}\right) . \quad$ IR: 3054, 3033, 2960, 2924, 2867, 2373, 2338, 1953, 1581, 1455, 1435, 1259, 1062, 999, 925, 826, 799, 731, $699 \mathrm{~cm}^{-1}$. HRMS $\left(\mathrm{FAB}^{+}\right.$) calcd for $\left[\mathrm{C}_{13} \mathrm{H}_{16} \mathrm{BNP}\right]^{+}$228.1113, Found 228.1118. Analysis of enantiomers by chiral HPLC (Chiralcel OJ Column, flow rate $1.1 \mathrm{~mL} / \mathrm{min}, 85: 15$ hexanes:i-PrOH, $\mathrm{T}_{\mathrm{r}}$ major 46.05 , minor $71.29 \mathrm{~min}$ ) determined the ee to $48 \%$.

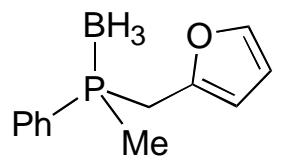

(2-methylfuryl)methylphenylphosphine borane. Flash chromatography (90:10 hexanes:EtOAc) furnished product as a yellow oil $(35 \mathrm{mg}, 80 \%) .{ }^{1} \mathrm{H} \mathrm{NMR}\left(\mathrm{CDCl}_{3}, 400\right.$ MHz) $\delta 7.61-7.42(\mathrm{~m}, 5 \mathrm{H}), 7.31(\mathrm{~m}, 1 \mathrm{H}), 6.29(\mathrm{~d}, J=2.8 \mathrm{~Hz}, 1 \mathrm{H}), 5.97(\mathrm{dd}, J=2.8,3.2 \mathrm{~Hz}$, $1 \mathrm{H}), 3.27(\mathrm{~d}, J=8.0 \mathrm{~Hz}, 2 \mathrm{H}), 1.61(\mathrm{~d}, J=10.0 \mathrm{~Hz}, 3 \mathrm{H}), 0.78(\mathrm{q}, J=89.6 \mathrm{~Hz}, 3 \mathrm{H}) .{ }^{13} \mathrm{C}\left\{{ }^{1} \mathrm{H}\right\}$ NMR $\left(\mathrm{CDCl}_{3}, 100 \mathrm{MHz}\right) \delta 146.9(\mathrm{~d}, J=9 \mathrm{~Hz}), 142.0,131.6(\mathrm{~d}, J=9 \mathrm{~Hz}), 131.5,128.6(\mathrm{~m})$, 110.7, $108.6(\mathrm{~d}, J=5 \mathrm{~Hz}), 28.5(\mathrm{~d}, J=33 \mathrm{~Hz}), 9.5(\mathrm{~d}, J=39 \mathrm{~Hz}) .{ }^{31} \mathrm{P}\left\{{ }^{1} \mathrm{H}\right\} \mathrm{NMR}\left(\mathrm{CDCl}_{3}, 162\right.$ MHz) $\delta 11.1\left(\mathrm{q}, J_{\mathrm{B}-\mathrm{P}}=65 \mathrm{~Hz}\right) . \quad$ IR: 3117, 3057, 2957, 2919, 2852, 2373, 2338, 2252, 1590, 
1502, 1437, 1416, 1257, 1147, 1118, 1062, 1010, 934, 900, 735, $692 \mathrm{~cm}^{-1}$. HRMS $\left(\mathrm{EI}^{+}\right)$calcd for $\left[\mathrm{C}_{12} \mathrm{H}_{15} \mathrm{BOP}\right]^{+}$217.0953, Found 217.0957. Analysis of enantiomers by chiral HPLC (Chiralcel OJ Column, flow rate $1.0 \mathrm{~mL} / \mathrm{min}, 98: 2$ hexanes:EtOH, $\mathrm{T}_{\mathrm{r}}$ minor 54.86, major 61.58 min) determined the ee to be $68 \%$.

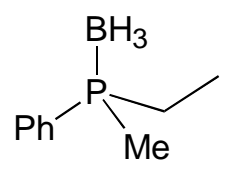

Ethylmethylphenylphosphine borane. Flash chromatography (70:30 hexanes: $\left.\mathrm{CH}_{2} \mathrm{Cl}_{2}\right)$ furnished product as a yellow oil $(23 \mathrm{mg}, 70 \%) .{ }^{1} \mathrm{H} \mathrm{NMR}\left(\mathrm{CDCl}_{3}, 400 \mathrm{MHz}\right) \delta$ 7.74-7.65 (m, 2H), $7.47(\mathrm{~m}, 3 \mathrm{H}), 1.83(\mathrm{~m}, 2 \mathrm{H}), 1.54(\mathrm{~d}, J=10.4 \mathrm{~Hz}, 3 \mathrm{H}), 1.09-0.39(\mathrm{~m}, 6 \mathrm{H}$, $\left.\mathrm{CH}_{3}+\mathrm{B} H_{3}\right) \cdot{ }^{13} \mathrm{C}\left\{{ }^{1} \mathrm{H}\right\} \mathrm{NMR}\left(\mathrm{CDCl}_{3}, 100 \mathrm{MHz}\right) \delta 132.1(\mathrm{~d}, J=8 \mathrm{~Hz}), 131.4(\mathrm{~d}, J=9 \mathrm{~Hz})$, 131.2, $128.8(\mathrm{~d}, J=9 \mathrm{~Hz}), 20.5(\mathrm{~d}, J=37 \mathrm{~Hz}), 10.2(\mathrm{~d}, J=38 \mathrm{~Hz}), 7.1 .{ }^{31} \mathrm{P}\left\{{ }^{1} \mathrm{H}\right\} \mathrm{NMR}\left(\mathrm{CDCl}_{3}\right.$, $162 \mathrm{MHz}) \delta 11.1\left(\mathrm{q}, J_{\mathrm{B}-\mathrm{P}}=63 \mathrm{~Hz}\right) . \quad$ IR: 3057, 2973, 2936, 2370, 2254, 1655, 1436, 1115, 1436, 1134, 1066, 902, 889, 743, 723, $692 \mathrm{~cm}^{-1}$. HRMS $\left(\mathrm{EI}^{+}\right)$calcd for $\left[\mathrm{C}_{9} \mathrm{H}_{15} \mathrm{BP}\right]^{+}$165.1003, Found 165.1004. Analysis of enantiomers by chiral HPLC (Chiralcel OJ Column, flow rate 0.9 $\mathrm{mL} / \mathrm{min}, 91: 9$ hexanes: $i-\mathrm{PrOH}, \mathrm{T}_{\mathrm{r}}$ minor 14.99 , major $17.20 \mathrm{~min}$ ) determined the ee to be $57 \%$. 


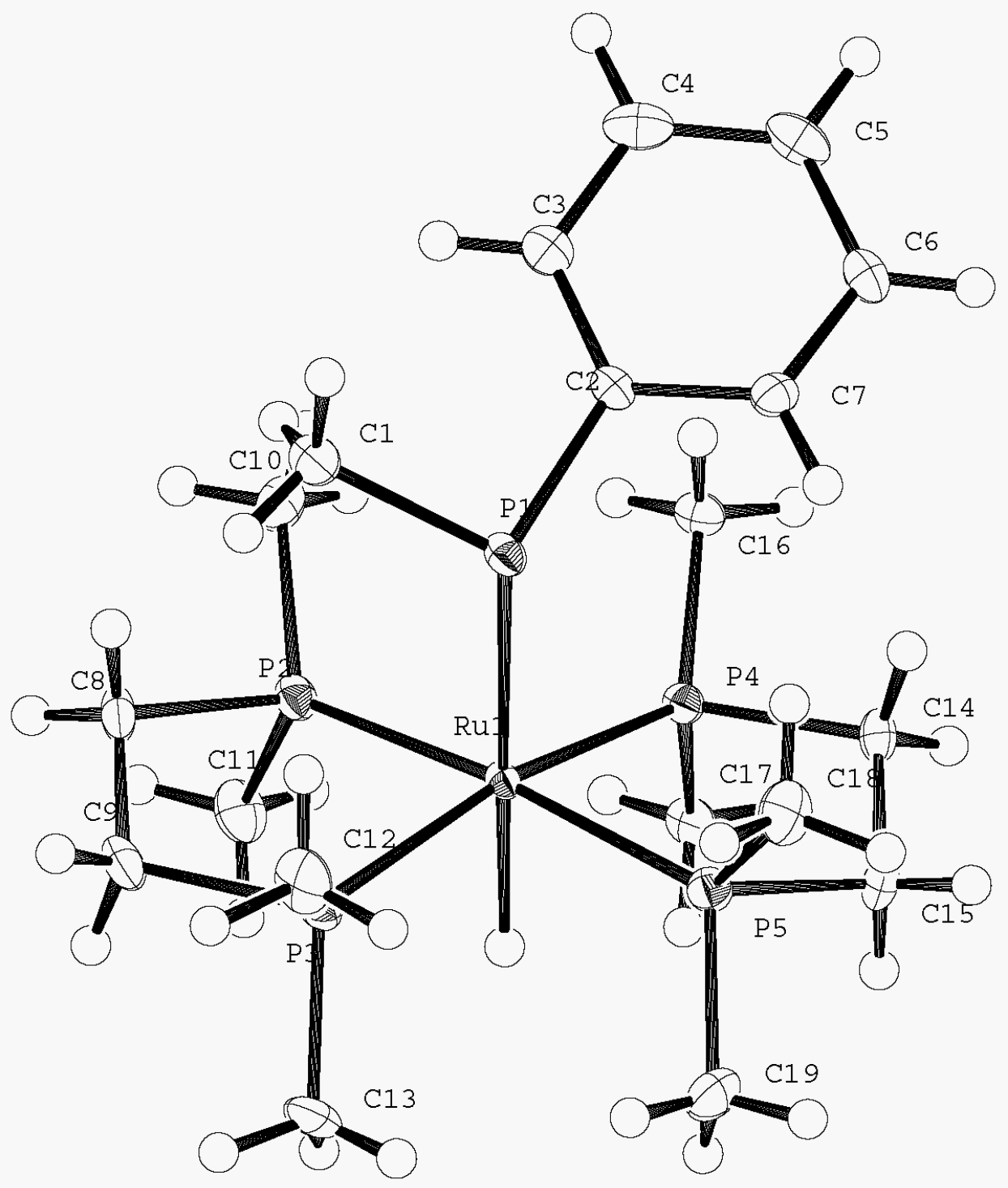

Figure $\boldsymbol{S}$-1. ORTEP of trans-(dmpe $)_{2} \mathrm{Ru}(\mathrm{H}) \mathrm{PMePh}(\mathbf{1})$. 


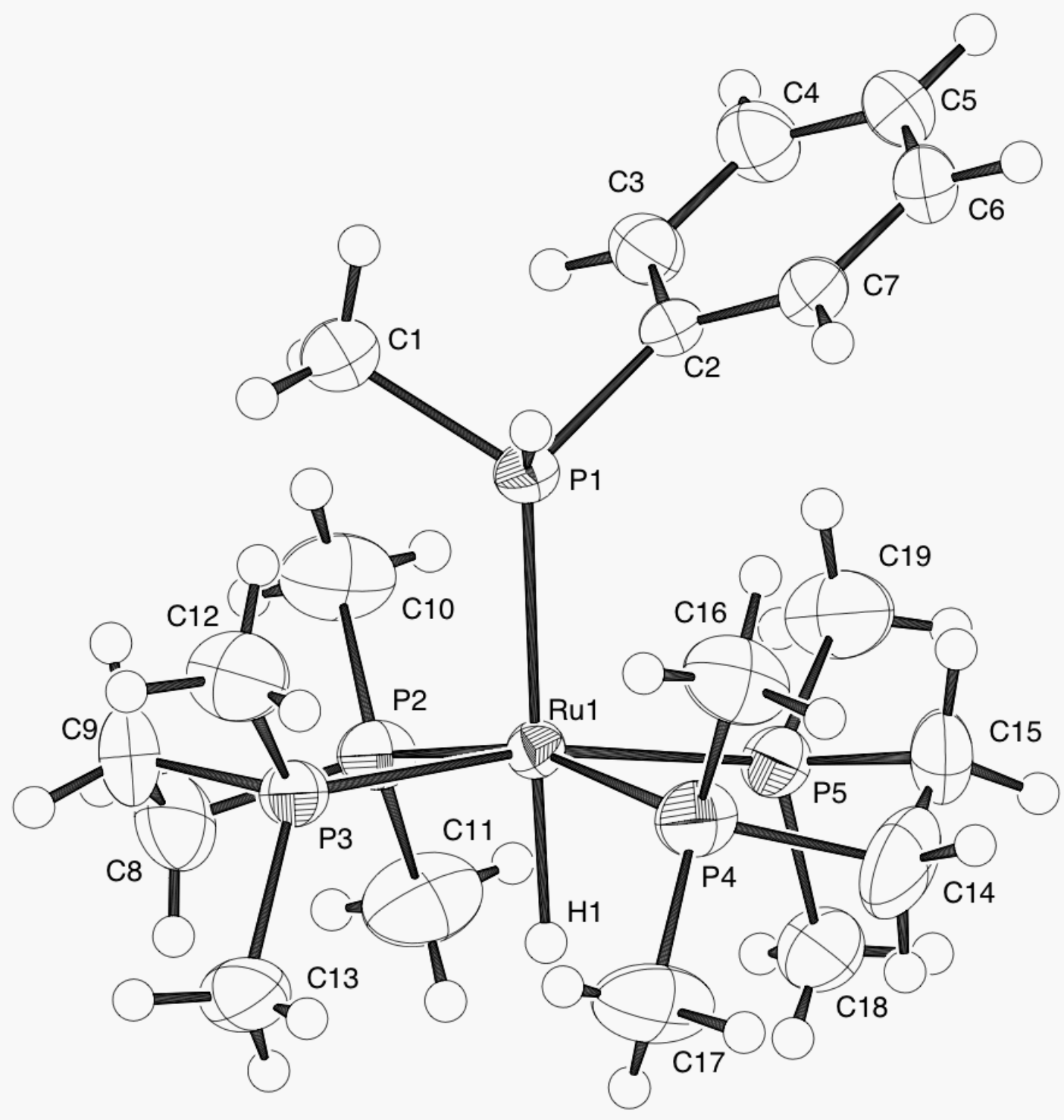

Figure S-2. ORTEP of cationic portion of $\left[\right.$ trans- $\left.(\mathrm{dmpe})_{2} \mathrm{Ru}(\mathrm{H}) \mathrm{P}(\mathrm{H}) \mathrm{MePh}\right]\left[\mathrm{BPh}_{4}\right]\left(\mathbf{2}-\mathbf{B P h} \mathbf{P h}_{\mathbf{4}}\right)$. 
General Experimental Details for X-Ray Structure Determinations. A fragment of a crystal of was mounted on a glass fiber using Paratone $\mathrm{N}$ hydrocarbon oil. All measurements were made on either a SMART ${ }^{6}$ CCD (1) or a Bruker $\operatorname{APEX}^{7}$ CCD (2-BPh $)$ area detector with graphite monochromated Mo-K $\alpha$ radiation. The data were collected at a temperature of 1: -153 $\pm 1{ }^{\circ} \mathrm{C}$; 2-BPh $4: 0 \pm 1{ }^{\circ} \mathrm{C}$. Frames corresponding to an arbitrary hemisphere of data were collected using $\omega$ scans of $0.3^{\circ}$ counted for a total of 10.0 seconds per frame. Data were integrated by the program $\mathrm{SAINT}^{8}$ to a maximum $2 \theta$ value of $\mathbf{1}: 49.4^{\circ} ; \mathbf{2}-\mathbf{B P h}_{\mathbf{4}}: 56.5^{\circ}$. The data were corrected for Lorentz and polarization effects. Data were analyzed for agreement and possible absorption using XPREP. ${ }^{9}$ An empirical absorption correction based on comparison of redundant and equivalent reflections was applied using $\operatorname{SADABS}^{10}(\mathbf{1}: \operatorname{Tmax}=1.00, \mathrm{Tmin}=$ 0.54; 2-BPh 4 : Tmax=1.00, Tmin=0.89). The structure was solved by direct methods ${ }^{11}$ and expanded using Fourier techniques. ${ }^{12}$ The non-hydrogen atoms were refined anisotropically in $\mathbf{1}$, while some were refined isotropically in $\mathbf{2}-\mathbf{B} \mathbf{P h}$. The final cycle of full-matrix least-squares

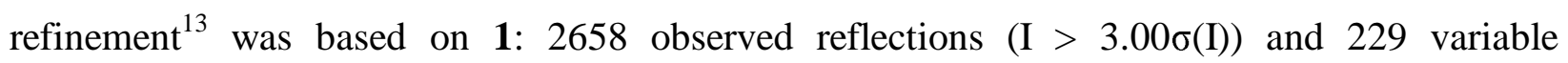
parameters; 2-BPh 4 : 8084 observed reflections $(\mathrm{I}>3.00 \sigma(\mathrm{I}))$ and 518 variable parameters The standard deviation of an observation of unit weight ${ }^{14}$ was 1: $1.29 ; \mathbf{2 - B P h}$ : 1.68 . The weighting scheme was based on counting statistics and included a factor $(\mathrm{p}=0.030)$ to downweight the intense reflections. Plots of $\Sigma \mathrm{w}(|\mathrm{Fo}|-|\mathrm{Fc}|)^{2}$ versus $|\mathrm{Fo}|$, reflection order in data collection, sin $\theta / \lambda$ and various classes of indices showed no unusual trends. The maximum and minimum peaks on the final difference Fourier map corresponded to $1: 0.89$ and -0.85 e- $/ \AA^{3} ; \mathbf{2}-\mathbf{B P h}_{\mathbf{4}}: 0.65$ and 0.26 e- $/ \AA^{3}$, respectively. Neutral atom scattering factors were taken from Cromer and Waber. ${ }^{15}$ Anomalous dispersion effects were included in Fcalc; ${ }^{16}$ the values for $\Delta \mathrm{f}^{\prime}$ and $\Delta \mathrm{f}^{\prime \prime}$ were those of Creagh and McAuley. ${ }^{17}$ The values for the mass attenuation coefficients are those of Creagh and Hubbel. ${ }^{18}$ All calculations were performed using the teXsan ${ }^{19}$ crystallographic software package of Molecular Structure Corporation. 
Table S-1. Crystal and Data Collection Parameters for trans-(dmpe)2Ru(H)P(Me)Ph (1).

A. Crystal Data

Empirical Formula

Formula Weight

Crystal Color, Habit

Crystal Dimensions

Crystal System

Lattice Type

Lattice Parameters

Space Group

$Z$ value

$D_{\text {calc }}$

$\mathrm{F}_{000}$

$\mu(\mathrm{MoK} \alpha)$

B. Intensity Measurements

Diffractometer

Radiation

Detector Position

Exposure Time

Scan Type

$2 \theta_{\max }$

No. of Reflections Measured

Corrections

C. Structure Solution and Refinement Structure Solution

Refinement

Function Minimized

Least Squares Weights

p-factor

Anomalous Dispersion

No. Observations $(I>3.00 \sigma(I))$

No. Variables

Reflection/Parameter Ratio

Residuals: R; Rw; Rall

Goodness of Fit Indicator

Max Shift/Error in Final Cycle

Maximum peak in Final Diff. Map

Minimum peak in Final Diff. Map
$\mathrm{P}_{5} \mathrm{C}_{19} \mathrm{H}_{41} \mathrm{Ru}$

525.47

yellow, needles

$0.16 \times 0.07 \times 0.07 \mathrm{~mm}$

monoclinic

Primitive

$a=9.4480(7) \AA$

$\mathrm{b}=15.478(1) \AA$

$\mathrm{C}=17.141(1) \AA$

$\beta=94.165(1)^{\circ}$

$\mathrm{V}=2500.0(3) \AA^{3}$

$\mathrm{P} 2{ }_{1} / \mathrm{c}(\# 14)$

4

$1.396 \mathrm{~g} / \mathrm{cm}^{3}$

1096.00

$9.50 \mathrm{~cm}^{-1}$

Bruker SMART CCD

$\operatorname{MoK} \alpha(\lambda=0.71069 \AA)$

graphite monochromated

$60.00 \mathrm{~mm}$

10.0 seconds per frame.

$\omega$ ( 0.3 degrees per frame)

$49.4^{\circ}$

Total: 11180

Unique: $2500\left(R_{\text {int }}=0.043\right)$

Lorentz-polarization

Absorption $(\operatorname{Tmax}=1.00 \mathrm{Tmin}=0.54)$

Direct Methods (SIR97)

Full-matrix least-squares

$\Sigma \mathrm{w}(|\mathrm{Fo}|-|\mathrm{Fc}|)^{2}$

$1 / \sigma^{2}(\mathrm{Fo})=4 \mathrm{Fo}^{2} / \sigma^{2}\left(\mathrm{Fo}^{2}\right)$

0.0300

All non-hydrogen atoms

2658

229

11.61

$0.033 ; 0.036 ; 0.058$

1.29

0.04

$0.89 \mathrm{e}^{-} / \AA^{3}$

$-0.85 \mathrm{e}^{-} / \AA^{3}$ 
Table S-2. Atomic coordinates and $B_{\text {iso }} / B_{\text {eq }}$ for trans- $(d m p e){ }_{2} R u(H) P(M e) P h(1)$.

\begin{tabular}{|c|c|c|c|c|}
\hline atom & $\mathbf{x}$ & $\mathbf{y}$ & $\mathbf{Z}$ & $B_{e q}$ \\
\hline $\mathrm{Ru}(1)$ & $-0.00300(5)$ & $0.00305(2)$ & $-0.00137(3)$ & $0.864(8)$ \\
\hline$P(1)^{\prime}$ & $0.1318(1)$ & $-0.12496(8)$ & $-0.04727(8)$ & $1.17(3)$ \\
\hline$P(2)$ & $0.0440(1)$ & $0.09047(8)$ & $-0.10435(8)$ & $1.27(3)$ \\
\hline$P(3)$ & $0.1980(1)$ & $0.06532(9)$ & $0.05740(8)$ & 1.41(3) \\
\hline$P(4)$ & $-0.2300(1)$ & $-0.03087(8)$ & $-0.04986(8)$ & $1.08(3)$ \\
\hline$P(5)$ & $-0.0636(1)$ & $-0.07046(9)$ & $0.10777(8)$ & $1.33(3)$ \\
\hline$C(1)$ & $0.2800(5)$ & $-0.0978(3)$ & $-0.1090(3)$ & $1.8(1)$ \\
\hline$C(2)$ & $0.0270(5)$ & $-0.1939(3)$ & $-0.1173(3)$ & $1.0(1)$ \\
\hline$C(3)$ & $0.0459(5)$ & $-0.2002(3)$ & $-0.1971(3)$ & $1.5(1)$ \\
\hline C(4) & $-0.0341(6)$ & $-0.2574(3)$ & $-0.2456(3)$ & $1.9(1)$ \\
\hline C(5) & $-0.1389(6)$ & $-0.3080(3)$ & $-0.2152(3)$ & $1.9(1)$ \\
\hline$C(6)$ & $-0.1609(5)$ & $-0.3024(3)$ & $-0.1359(3)$ & $1.5(1)$ \\
\hline$C(7)$ & $-0.0767(5)$ & $-0.2480(3)$ & $-0.0885(3)$ & $1.3(1)$ \\
\hline$C(8)$ & $0.2331(5)$ & $0.1226(3)$ & $-0.0962(3)$ & $1.6(1)$ \\
\hline $\mathrm{C}(9)$ & $0.2790(5)$ & $0.1409(3)$ & $-0.0104(3)$ & $1.6(1)$ \\
\hline$C(10)$ & $0.0170(6)$ & $0.0571(3)$ & $-0.2065(3)$ & $1.9(1)$ \\
\hline$C(11)$ & $-0.0402(6)$ & $0.1974(3)$ & $-0.1100(3)$ & $2.0(1)$ \\
\hline$C(12)$ & $0.3469(5)$ & $-0.0002(4)$ & $0.0976(3)$ & $2.2(1)$ \\
\hline$C(13)$ & $0.1803(6)$ & $0.1379(4)$ & $0.1403(3)$ & $2.4(1)$ \\
\hline$C(14)$ & $-0.3073(5)$ & $-0.1109(3)$ & $0.0141(3)$ & $1.3(1)$ \\
\hline$C(15)$ & $-0.2570(5)$ & $-0.0932(3)$ & 0.0994(3) & $1.6(1)$ \\
\hline$C(16)$ & $-0.2825(5)$ & $-0.0718(3)$ & $-0.1471(3)$ & $1.4(1)$ \\
\hline$C(17)$ & $-0.3585(5)$ & $0.0571(3)$ & $-0.0451(3)$ & $1.5(1)$ \\
\hline$C(18)$ & $0.0132(6)$ & $-0.1760(3)$ & $0.1349(3)$ & $2.0(1)$ \\
\hline$C(19)$ & $-0.0502(6)$ & $-0.0197(4)$ & $0.2045(3)$ & $2.2(1)$ \\
\hline$H(1)$ & 0.3266 & -0.1493 & -0.1230 & 2.1238 \\
\hline $\mathrm{H}(2)$ & 0.2432 & -0.0690 & -0.1551 & 2.1238 \\
\hline $\mathrm{H}(3)$ & 0.3458 & -0.0611 & -0.0806 & 2.1238 \\
\hline $\mathrm{H}(4)$ & 0.1149 & -0.1647 & -0.2190 & 1.7446 \\
\hline$H(5)$ & -0.0170 & -0.2618 & -0.2994 & 2.2750 \\
\hline$H(6)$ & -0.1948 & -0.3460 & -0.2482 & 2.2409 \\
\hline $\mathrm{H}(7)$ & -0.2332 & -0.3358 & -0.1146 & 1.8432 \\
\hline $\mathrm{H}(8)$ & -0.0895 & -0.2472 & -0.0340 & 1.6145 \\
\hline $\mathrm{H}(9)$ & 0.2892 & 0.0772 & -0.1148 & 1.9303 \\
\hline$H(10)$ & 0.2453 & 0.1731 & -0.1264 & 1.9303 \\
\hline$H(11)$ & 0.2517 & 0.1981 & 0.0017 & 1.9643 \\
\hline$H(12)$ & 0.3793 & 0.1358 & -0.0032 & 1.9643 \\
\hline$H(13)$ & -0.0818 & 0.0556 & -0.2215 & 2.3227 \\
\hline $\mathrm{H}(14)$ & 0.0623 & 0.0970 & -0.2386 & 2.3227 \\
\hline
\end{tabular}




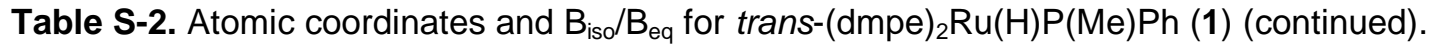

\begin{tabular}{|c|c|c|c|c|}
\hline atom & $\mathbf{x}$ & $\mathbf{y}$ & $\mathbf{Z}$ & $B_{e q}$ \\
\hline $\mathrm{H}(15)$ & 0.0563 & 0.0012 & -0.2125 & 2.3227 \\
\hline$H(16)$ & -0.0348 & 0.2230 & -0.0595 & 2.3873 \\
\hline $\mathrm{H}(17)$ & 0.0076 & 0.2331 & -0.1447 & 2.3873 \\
\hline$H(18)$ & -0.1369 & 0.1915 & -0.1286 & 2.3873 \\
\hline $\mathrm{H}(19)$ & 0.3211 & -0.0288 & 0.1435 & 2.5933 \\
\hline $\mathrm{H}(20)$ & 0.3705 & -0.0419 & 0.0599 & 2.5933 \\
\hline $\mathrm{H}(21)$ & 0.4266 & 0.0360 & 0.1101 & 2.5933 \\
\hline $\mathrm{H}(22)$ & 0.2665 & 0.1688 & 0.1509 & 2.9208 \\
\hline $\mathrm{H}(23)$ & 0.1052 & 0.1775 & 0.1280 & 2.9208 \\
\hline $\mathrm{H}(24)$ & 0.1602 & 0.1051 & 0.1850 & 2.9208 \\
\hline $\mathrm{H}(25)$ & -0.4079 & -0.1072 & 0.0080 & 1.6079 \\
\hline $\mathrm{H}(26)$ & -0.2782 & -0.1672 & -0.0000 & 1.6079 \\
\hline $\mathrm{H}(27)$ & -0.2758 & -0.1423 & 0.1302 & 1.9730 \\
\hline $\mathrm{H}(28)$ & -0.3066 & -0.0447 & 0.1177 & 1.9730 \\
\hline $\mathrm{H}(29)$ & -0.3787 & -0.0901 & -0.1490 & 1.6666 \\
\hline $\mathrm{H}(30)$ & -0.2726 & -0.0273 & -0.1845 & 1.6666 \\
\hline$H(31)$ & -0.2238 & -0.1192 & -0.1585 & 1.6666 \\
\hline $\mathrm{H}(32)$ & -0.3444 & 0.0856 & 0.0040 & 1.7891 \\
\hline $\mathrm{H}(33)$ & -0.3459 & 0.0972 & -0.0860 & 1.7891 \\
\hline $\mathrm{H}(34)$ & -0.4520 & 0.0343 & -0.0507 & 1.7891 \\
\hline $\mathrm{H}(35)$ & -0.0430 & -0.2033 & 0.1716 & 2.4437 \\
\hline $\mathrm{H}(36)$ & 0.0153 & -0.2111 & 0.0896 & 2.4437 \\
\hline $\mathrm{H}(37)$ & 0.1070 & -0.1683 & 0.1577 & 2.4437 \\
\hline $\mathrm{H}(38)$ & 0.0455 & -0.0038 & 0.2179 & 2.6350 \\
\hline$H(39)$ & -0.1086 & 0.0303 & 0.2036 & 2.6350 \\
\hline$H(40)$ & -0.0807 & -0.0594 & 0.2421 & 2.6350 \\
\hline $\mathrm{H}(41)$ & $-0.082(5)$ & $0.086(3)$ & $0.042(3)$ & 2.2982 \\
\hline
\end{tabular}


Table S-3. Anisotropic Displacement Parameters for trans-(dmpe $)_{2} \mathrm{Ru}(\mathrm{H}) \mathrm{P}(\mathrm{Me}) \mathrm{Ph}(\mathbf{1})$.

\begin{tabular}{lllllll} 
atom & $\mathbf{U}_{11}$ & $\mathbf{U}_{22}$ & $\mathbf{U}_{33}$ & $\mathbf{U}_{12}$ & \multicolumn{1}{c}{$\mathbf{U}_{13}$} & \multicolumn{1}{c}{$\mathbf{U}_{\mathbf{2 3}}$} \\
\hline $\mathrm{Ru}(1)$ & $0.0094(2)$ & $0.0097(2)$ & $0.0137(2)$ & $-0.0004(2)$ & $0.0003(1)$ & $-0.0009(2)$ \\
$\mathrm{P}(1)$ & $0.0136(7)$ & $0.0115(7)$ & $0.0191(8)$ & $-0.0002(6)$ & $0.0003(6)$ & $-0.0014(6)$ \\
$\mathrm{P}(2)$ & $0.0163(7)$ & $0.0124(7)$ & $0.0196(8)$ & $-0.0007(6)$ & $0.0017(6)$ & $-0.0001(6)$ \\
$\mathrm{P}(3)$ & $0.0132(7)$ & $0.0179(8)$ & $0.0223(8)$ & $-0.0029(6)$ & $-0.0005(6)$ & $-0.0045(7)$ \\
$\mathrm{P}(4)$ & $0.0112(7)$ & $0.0139(7)$ & $0.0158(8)$ & $0.0002(6)$ & $0.0008(6)$ & $-0.0019(6)$ \\
$\mathrm{P}(5)$ & $0.0182(8)$ & $0.0172(7)$ & $0.0152(8)$ & $-0.0011(6)$ & $0.0004(6)$ & $0.0002(6)$ \\
$\mathrm{C}(1)$ & $0.013(3)$ & $0.021(3)$ & $0.033(4)$ & $0.001(2)$ & $0.005(3)$ & $-0.008(3)$ \\
$\mathrm{C}(2)$ & $0.012(3)$ & $0.009(3)$ & $0.018(3)$ & $0.004(2)$ & $-0.002(2)$ & $0.001(2)$ \\
$\mathrm{C}(3)$ & $0.020(3)$ & $0.014(3)$ & $0.022(3)$ & $0.004(2)$ & $-0.000(2)$ & $0.001(2)$ \\
$\mathrm{C}(4)$ & $0.025(3)$ & $0.024(3)$ & $0.022(3)$ & $0.012(3)$ & $-0.004(3)$ & $-0.005(3)$ \\
$\mathrm{C}(5)$ & $0.023(3)$ & $0.019(3)$ & $0.026(4)$ & $0.003(2)$ & $-0.012(3)$ & $-0.004(3)$ \\
$\mathrm{C}(6)$ & $0.016(3)$ & $0.016(3)$ & $0.026(3)$ & $-0.002(2)$ & $-0.002(3)$ & $-0.000(2)$ \\
$\mathrm{C}(7)$ & $0.021(3)$ & $0.014(3)$ & $0.017(3)$ & $0.003(2)$ & $0.003(2)$ & $0.001(2)$ \\
$\mathrm{C}(8)$ & $0.016(3)$ & $0.016(3)$ & $0.030(4)$ & $-0.005(2)$ & $0.007(3)$ & $0.002(2)$ \\
$\mathrm{C}(9)$ & $0.017(3)$ & $0.013(3)$ & $0.032(4)$ & $-0.003(2)$ & $0.002(3)$ & $-0.002(2)$ \\
$\mathrm{C}(10)$ & $0.029(3)$ & $0.027(3)$ & $0.018(3)$ & $-0.005(3)$ & $0.005(3)$ & $0.003(3)$ \\
$\mathrm{C}(11)$ & $0.025(3)$ & $0.016(3)$ & $0.034(4)$ & $0.001(2)$ & $0.001(3)$ & $0.005(3)$ \\
$\mathrm{C}(12)$ & $0.015(3)$ & $0.038(3)$ & $0.029(4)$ & $-0.004(3)$ & $-0.008(3)$ & $0.001(3)$ \\
$\mathrm{C}(13)$ & $0.030(3)$ & $0.033(4)$ & $0.030(4)$ & $-0.013(3)$ & $0.006(3)$ & $-0.020(3)$ \\
$\mathrm{C}(14)$ & $0.010(3)$ & $0.019(3)$ & $0.023(3)$ & $-0.002(2)$ & $0.002(2)$ & $0.003(2)$ \\
$\mathrm{C}(15)$ & $0.018(3)$ & $0.023(3)$ & $0.023(3)$ & $-0.005(2)$ & $0.008(3)$ & $0.002(3)$ \\
$\mathrm{C}(16)$ & $0.016(3)$ & $0.018(3)$ & $0.018(3)$ & $0.002(2)$ & $-0.001(2)$ & $-0.003(2)$ \\
$\mathrm{C}(17)$ & $0.010(3)$ & $0.022(3)$ & $0.024(3)$ & $0.001(2)$ & $-0.001(2)$ & $-0.004(3)$ \\
$\mathrm{C}(18)$ & $0.030(3)$ & $0.022(3)$ & $0.026(4)$ & $0.002(3)$ & $0.003(3)$ & $0.010(3)$ \\
$\mathrm{C}(19)$ & $0.031(3)$ & $0.036(4)$ & $0.017(3)$ & $-0.005(3)$ & $0.008(3)$ & $-0.001(3)$ \\
\hline $\mathrm{Th}$ & $0.01(3)$ & & &
\end{tabular}

The general temperature factor expression:

$\exp \left(-2 \pi^{2}\left(a^{* 2} U_{11} h^{2}+b^{* 2} U_{22} k^{2}+c^{* 2} U_{33} l^{2}+2 a * b * U_{12} h k+2 a^{*} c^{*} U_{13} h l+2 b^{*} c^{*} U_{23} k l\right)\right)$ 
Table S-4. Bond Lengths $(\AA)$ for trans-(dmpe ${ }_{2} R u(H) P(M e) P h(1)$.

\begin{tabular}{llllll} 
atom & atom & distance & atom & atom & distance \\
\hline Ru1 & P1 & $2.513(1)$ & Ru1 & P2 & $2.293(1)$ \\
Ru1 & P3 & $2.296(1)$ & Ru1 & P4 & $2.304(1)$ \\
Ru1 & P5 & $2.297(1)$ & P1 & C1 & $1.864(5)$ \\
P1 & C2 & $1.840(5)$ & P2 & C8 & $1.850(5)$ \\
P2 & C10 & $1.826(5)$ & P2 & C11 & $1.836(5)$ \\
P3 & C9 & $1.853(5)$ & P3 & C12 & $1.829(5)$ \\
P3 & C13 & $1.828(5)$ & P4 & C14 & $1.839(5)$ \\
P4 & C16 & $1.818(5)$ & P4 & C17 & $1.830(5)$ \\
P5 & C15 & $1.856(5)$ & P5 & C18 & $1.834(5)$ \\
P5 & C19 & $1.830(5)$ & C2 & C3 & $1.396(7)$ \\
C2 & C7 & $1.406(6)$ & C3 & C4 & $1.399(7)$ \\
C4 & C5 & $1.393(7)$ & C5 & C6 & $1.392(7)$ \\
C6 & C7 & $1.381(7)$ & C8 & C9 & $1.528(7)$ \\
C14 & C15 & $1.529(7)$ & Ru1 & H41 & $1.68(4)$ \\
\hline
\end{tabular}


Table S-5. Bond Angles $\left({ }^{\circ}\right)$ for trans-(dmpe ${ }_{2} \mathrm{Ru}(\mathrm{H}) \mathrm{P}(\mathrm{Me}) \mathrm{Ph}(\mathbf{1})$.

\begin{tabular}{llllllll} 
atom & atom & atom & angle & atom & atom & atom & angle \\
\hline P1 & Ru1 & P2 & $95.35(5)$ & P1 & Ru1 & P3 & $92.67(5)$ \\
P1 & Ru1 & P4 & $100.79(4)$ & P1 & Ru1 & P5 & $91.52(5)$ \\
P2 & Ru1 & P3 & $83.67(5)$ & P2 & Ru1 & P4 & $94.83(5)$ \\
P2 & Ru1 & P5 & $173.12(5)$ & P3 & Ru1 & P4 & $166.54(5)$ \\
P3 & Ru1 & P5 & $95.61(5)$ & P4 & Ru1 & P5 & $84.27(5)$ \\
Ru1 & P1 & C1 & $114.8(2)$ & Ru1 & P1 & C2 & $113.6(2)$ \\
C1 & P1 & C2 & $98.7(2)$ & Ru1 & P2 & C8 & $109.9(2)$ \\
Ru1 & P2 & C10 & $123.4(2)$ & Ru1 & P2 & C11 & $117.7(2)$ \\
C8 & P2 & C10 & $102.5(2)$ & C8 & P2 & C11 & $100.0(2)$ \\
C10 & P2 & C11 & $100.1(2)$ & Ru1 & P3 & C9 & $110.8(2)$ \\
Ru1 & P3 & C12 & $121.4(2)$ & Ru1 & P3 & C13 & $118.7(2)$ \\
C9 & P3 & C12 & $104.1(2)$ & C9 & P3 & C13 & $99.5(2)$ \\
C12 & P3 & C13 & $99.2(3)$ & Ru1 & P4 & C14 & $109.7(2)$ \\
Ru1 & P4 & C16 & $126.5(2)$ & Ru1 & P4 & C17 & $114.7(2)$ \\
C14 & P4 & C16 & $102.6(2)$ & C14 & P4 & C17 & $100.4(2)$ \\
C16 & P4 & C17 & $99.3(2)$ & Ru1 & P5 & C15 & $109.5(2)$ \\
Ru1 & P5 & C18 & $122.0(2)$ & Ru1 & P5 & C19 & $121.3(2)$ \\
C15 & P5 & C18 & $102.7(2)$ & C15 & P5 & C19 & $98.9(2)$ \\
C18 & P5 & C19 & $98.8(3)$ & P1 & C2 & C3 & $125.3(4)$ \\
P1 & C2 & C7 & $118.2(4)$ & C3 & C2 & C7 & $116.4(4)$ \\
C2 & C3 & C4 & $121.7(5)$ & C3 & C4 & C5 & $120.1(5)$ \\
C4 & C5 & C6 & $119.4(5)$ & C5 & C6 & C7 & $119.5(5)$ \\
C2 & C7 & C6 & $122.9(5)$ & P2 & C8 & C9 & $109.1(3)$ \\
P3 & C9 & C8 & $112.6(3)$ & P4 & C14 & C15 & $109.9(3)$ \\
P5 & C15 & C14 & $110.1(3)$ & P2 & Ru1 & H41 & $90(1)$ \\
P1 & Ru1 & H41 & $172(1)$ & P4 & Ru1 & H41 & $84(1)$ \\
P3 & Ru1 & H41 & $82(1)$ & P5 & Ru1 & H41 & $82(1)$ \\
\hline
\end{tabular}


Table S-6. Torsion Angles $\left({ }^{\circ}\right)$ for trans-(dmpe $)_{2} \mathrm{Ru}(\mathrm{H}) \mathrm{P}(\mathrm{Me}) \mathrm{Ph}(\mathbf{1})$.

\begin{tabular}{|c|c|c|c|c|c|c|c|c|c|}
\hline atom & & & & & & 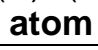 & & & \\
\hline Ru1 & $\mathrm{P} 1$ & C2 & C3 & 108.3(4) & Ru1 & $\mathrm{P} 1$ & $\mathrm{C} 2$ & C7 & $-74.9(4)$ \\
\hline Ru1 & P2 & C8 & C9 & $-39.5(4)$ & Ru1 & P3 & C9 & C8 & $-21.1(4)$ \\
\hline Ru1 & P4 & C14 & C15 & $35.9(4)$ & Ru1 & P5 & C15 & C14 & $34.9(4)$ \\
\hline P1 & Ru1 & P2 & C8 & $-71.0(2)$ & P1 & Ru1 & P2 & C10 & 49.9(2) \\
\hline P1 & Ru1 & P2 & C11 & $175.5(2)$ & P1 & Ru1 & P3 & C9 & $92.6(2)$ \\
\hline P1 & Ru1 & P3 & C12 & $-30.0(2)$ & P1 & Ru1 & P3 & C13 & $-153.2(2)$ \\
\hline P1 & Ru1 & P4 & C14 & $79.1(2)$ & P1 & Ru1 & P4 & C16 & $-44.6(2)$ \\
\hline P1 & Ru1 & P4 & C17 & $-168.8(2)$ & P1 & Ru1 & P5 & C15 & $-110.9(2)$ \\
\hline P1 & Ru1 & P5 & C18 & 8.9(2) & P1 & Ru1 & P5 & C19 & $135.1(2)$ \\
\hline P1 & C2 & C3 & C4 & $176.9(4)$ & P1 & C2 & $\mathrm{C} 7$ & C6 & $-179.8(4)$ \\
\hline P2 & Ru1 & P1 & C1 & $27.1(2)$ & P2 & Ru1 & P1 & C2 & $-85.5(2)$ \\
\hline P2 & Ru1 & P3 & C9 & $-2.5(2)$ & P2 & Ru1 & P3 & C12 & $-125.1(2)$ \\
\hline P2 & Ru1 & P3 & C13 & $111.7(2)$ & P2 & Ru1 & P4 & C14 & $175.4(2)$ \\
\hline P2 & Ru1 & P4 & C16 & $51.8(2)$ & P2 & Ru1 & P4 & C17 & $-72.4(2)$ \\
\hline P2 & Ru1 & P5 & C15 & 72.6(5) & P2 & Ru1 & P5 & C18 & $-167.6(4)$ \\
\hline P2 & Ru1 & P5 & C19 & $-41.4(5)$ & P2 & C8 & C9 & P3 & 37.8(4) \\
\hline P3 & Ru1 & P1 & C1 & $-56.8(2)$ & P3 & Ru1 & P1 & $\mathrm{C} 2$ & $-169.4(2)$ \\
\hline P3 & Ru1 & P2 & C8 & 21.1(2) & P3 & Ru1 & P2 & C10 & $142.0(2)$ \\
\hline P3 & Ru1 & P2 & C11 & $-92.4(2)$ & P3 & Ru1 & P4 & C14 & $-101.6(3)$ \\
\hline P3 & Ru1 & P4 & C16 & $134.8(3)$ & P3 & Ru1 & P4 & C17 & 10.5(3) \\
\hline P3 & Ru1 & P5 & C15 & $156.3(2)$ & P3 & Ru1 & P5 & C18 & $-84.0(2)$ \\
\hline P3 & Ru1 & P5 & C19 & $42.3(2)$ & P4 & Ru1 & P1 & C1 & $123.0(2)$ \\
\hline P4 & Ru1 & P1 & C2 & $10.5(2)$ & P4 & Ru1 & P2 & C8 & $-172.3(2)$ \\
\hline P4 & Ru1 & P2 & C10 & -51. & P4 & Ru1 & P2 & C11 & $74.1(2)$ \\
\hline P4 & Ru1 & P3 & C9 & $-86.8(3)$ & P4 & Ru1 & P3 & C12 & $150.6(3)$ \\
\hline P4 & Ru1 & P3 & C13 & $27.4(3)$ & P4 & Ru1 & P5 & C15 & $-10.2(2)$ \\
\hline P4 & Ru1 & P5 & C18 & $109.6(2)$ & P4 & Ru1 & P5 & C19 & $-124.2(2)$ \\
\hline P4 & C14 & C15 & P5 & $-44.6(4)$ & P5 & Ru1 & P1 & C1 & $-152.5(2)$ \\
\hline P5 & Ru1 & P1 & C2 & $94.9(2)$ & P5 & Ru1 & P2 & C8 & $105.5(5)$ \\
\hline P5 & Ru1 & P2 & C10 & $-133.6(4)$ & P5 & Ru1 & P2 & C11 & $-8.1(5)$ \\
\hline P5 & Ru1 & P3 & C9 & 6(2) & P5 & Ru1 & P3 & C12 & $61.8(2)$ \\
\hline P5 & Ru1 & P3 & C13 & & P5 & Ru1 & P4 & C1 & $-11.4(2)$ \\
\hline P5 & Ru1 & P4 & C16 & $0(2)$ & P5 & Ru1 & P4 & C17 & $100.7(2)$ \\
\hline C1 & P1 & $\mathrm{C} 2$ & C3 & $-13.6(5)$ & C1 & P1 & C2 & C7 & $163.2(4)$ \\
\hline $\mathrm{C} 2$ & C3 & C4 & C5 & $2.0(8)$ & C2 & C7 & C6 & C5 & $3.4(8)$ \\
\hline C3 & C2 & C7 & C6 & $-2.7(7)$ & C3 & C4 & C5 & C6 & $-1.4(8)$ \\
\hline C4 & C3 & C2 & C7 & $0.0(7)$ & C4 & C5 & C6 & C7 & $-1.2(8)$ \\
\hline C8 & C9 & P3 & C12 & 111.1(4) & C8 & C9 & P3 & C13 & $-146.8(4)$ \\
\hline C9 & C8 & P2 & C10 & $-172.3(3)$ & C9 & C8 & P2 & C11 & $85.0(4)$ \\
\hline C14 & C15 & P5 & C18 & $-96.1(4)$ & C14 & C15 & P5 & C19 & $162.8(4)$ \\
\hline C15 & C14 & P4 & C16 & $172.6(3)$ & C15 & C14 & P4 & C17 & $-85.2(4)$ \\
\hline
\end{tabular}


Table S-7. Crystal and Data Collection Parameters for [trans-(dmpe)2Ru(H)PH(Me)Ph][BPh $\left.{ }_{4}\right]\left(2-\mathbf{B P h}_{4}\right)$.

A. Crystal Data

Empirical Formula

Formula Weight

Crystal Color, Habit

Crystal Dimensions

Crystal System

Lattice Type

Lattice Parameters

Space Group

$Z$ value

$D_{\text {calc }}$

$\mathrm{F}_{000}$

$\mu(\mathrm{MoK} \alpha)$

B. Intensity Measurements

Diffractometer

Radiation

Detector Position

Exposure Time

Scan Type

$2 \theta_{\max }$

No. of Reflections Measured

Corrections

C. Structure Solution and Refinement Structure Solution

Refinement

Function Minimized

Least Squares Weights

p-factor

Anomalous Dispersion

No. Observations $(I>3.00 \sigma(I))$

No. Variables

Reflection/Parameter Ratio

Residuals: R; Rw; Rall

Goodness of Fit Indicator

Max Shift/Error in Final Cycle

Maximum peak in Final Diff. Map

Minimum peak in Final Diff. Map

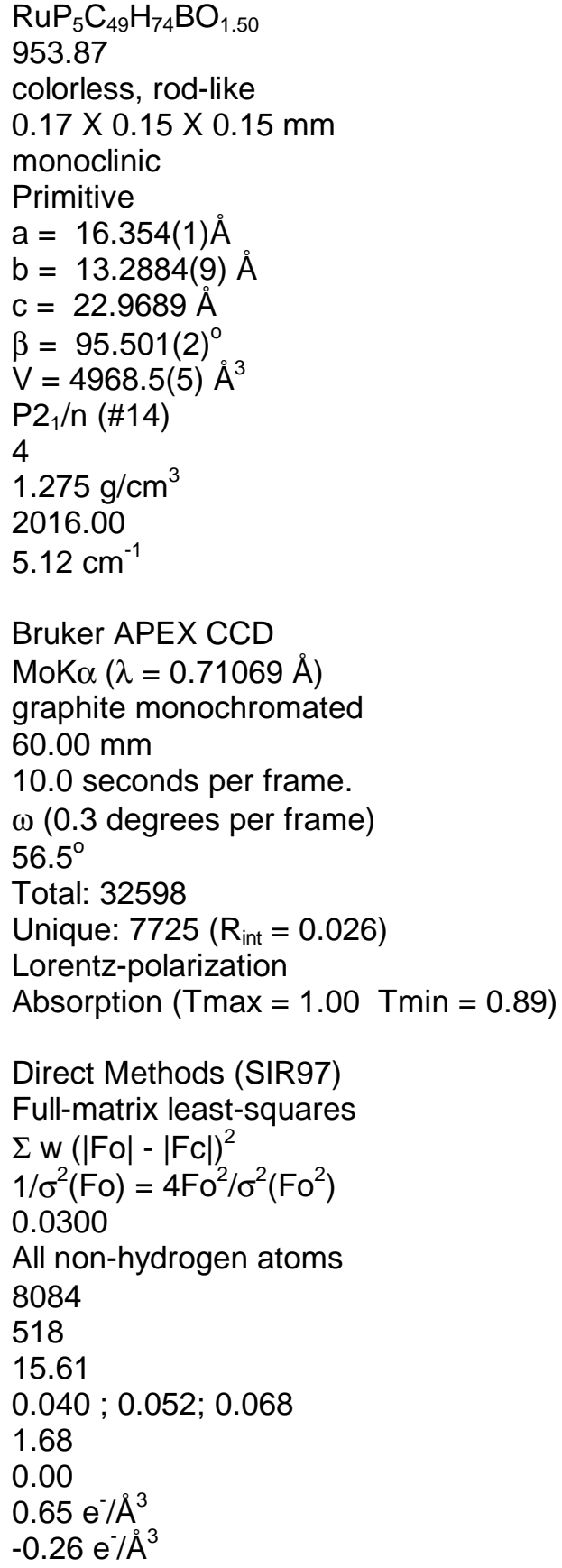


Table S-8. Atomic coordinates and $\mathrm{B}_{\text {iso }} / \mathrm{B}_{\text {eq }}$ and occupancy for [trans-(dmpe)2Ru(H)PH(Me)Ph][BPh $]$ (2$\mathrm{BPh}_{4}$ ).

\begin{tabular}{llllll} 
atom & $\mathbf{x}$ & $\mathbf{y}$ & $\mathbf{z}$ & $\mathbf{B}_{\text {eq }}$ & occupancy \\
\hline Ru1 & $0.52803(1)$ & $0.25013(2)$ & $0.232834(9)$ & $2.037(5)$ & \\
P1 & $0.64286(5)$ & $0.35114(6)$ & $0.22167(4)$ & $2.49(2)$ & \\
P2 & $0.46755(6)$ & $0.25320(6)$ & $0.13739(4)$ & $3.03(2)$ & \\
P3 & $0.44596(5)$ & $0.38946(6)$ & $0.24411(4)$ & $2.94(2)$ & \\
P4 & $0.55739(6)$ & $0.22107(7)$ & $0.33187(4)$ & $3.56(2)$ & \\
P5 & $0.58585(6)$ & $0.09201(6)$ & $0.22364(4)$ & $3.40(2)$ & \\
O1 & $0.9035(4)$ & $0.6865(5)$ & $-0.0223(2)$ & $13.0(2)$ & $1 / 2$ \\
O2 & $1.0123(5)$ & $0.0647(7)$ & $-0.0563(3)$ & $9.1(3)$ & \\
C1 & $0.6360(2)$ & $0.4636(3)$ & $0.1742(2)$ & $4.45(9)$ & \\
C2 & $0.7415(2)$ & $0.3023(2)$ & $0.2033(1)$ & $2.35(6)$ & \\
C3 & $0.7592(2)$ & $0.2926(3)$ & $0.1458(1)$ & $3.29(7)$ & \\
C4 & $0.8334(2)$ & $0.2535(3)$ & $0.1322(2)$ & $3.87(8)$ & \\
C5 & $0.8923(2)$ & $0.2239(3)$ & $0.1763(2)$ & $3.78(8)$ & \\
C6 & $0.8765(2)$ & $0.2333(2)$ & $0.2338(2)$ & $3.43(8)$ & \\
C7 & $0.8021(2)$ & $0.2721(2)$ & $0.2469(1)$ & $2.86(7)$ & \\
C8 & $0.3839(3)$ & $0.3447(3)$ & $0.1331(2)$ & $5.2(1)$ & \\
C9 & $0.4062(3)$ & $0.4336(3)$ & $0.1711(2)$ & $5.3(1)$ & \\
C10 & $0.5237(3)$ & $0.2868(4)$ & $0.0758(2)$ & $5.4(1)$ & \\
C11 & $0.4129(3)$ & $0.1428(3)$ & $0.1083(2)$ & $6.0(1)$ & \\
C12 & $0.4871(3)$ & $0.5025(3)$ & $0.2803(2)$ & $5.6(1)$ & \\
C13 & $0.3518(3)$ & $0.3753(3)$ & $0.2793(2)$ & $5.6(1)$ & \\
C14 & $0.5949(3)$ & $0.0917(3)$ & $0.3424(2)$ & $6.2(1)$ & \\
C15 & $0.6440(3)$ & $0.0616(3)$ & $0.2942(2)$ & $5.4(1)$ & \\
C16 & $0.6350(2)$ & $0.2929(3)$ & $0.3768(2)$ & $4.89(10)$ \\
C17 & $0.4738(3)$ & $0.2211(4)$ & $0.3791(2)$ & $6.0(1)$ & \\
C18 & $0.5158(3)$ & $-0.0148(3)$ & $0.2150(2)$ & $5.4(1)$ & \\
C19 & $0.6580(3)$ & $0.0606(3)$ & $0.1712(2)$ & $6.1(1)$ & \\
C20 & $0.5511(2)$ & $0.2258(2)$ & $-0.0818(1)$ & $2.38(6)$ & \\
C21 & $0.4887(2)$ & $0.2907(2)$ & $-0.1067(1)$ & $2.86(7)$ & \\
C22 & $0.4066(2)$ & $0.2731(2)$ & $-0.1045(1)$ & $3.18(7)$ & \\
C23 & $0.3805(2)$ & $0.1878(3)$ & $-0.0772(1)$ & $3.61(8)$ & \\
C24 & $0.4383(2)$ & $0.1205(2)$ & $-0.0534(1)$ & $3.34(8)$ & \\
C25 & $0.5216(2)$ & $0.1400(2)$ & $-0.0560(1)$ & $2.66(7)$ & \\
C26 & $0.6695(2)$ & $0.2827(2)$ & $-0.1487(1)$ & $2.60(6)$ & \\
C27 & $0.6246(2)$ & $0.2417(2)$ & $-0.1985(1)$ & $3.53(8)$ & \\
C28 & $0.6428(3)$ & $0.2639(3)$ & $-0.2553(2)$ & $4.43(10)$ & \\
C29 & $0.7067(3)$ & $0.3273(3)$ & $-0.2646(2)$ & $4.6(1)$ & \\
C30 & $0.7523(2)$ & $0.3684(3)$ & $-0.2173(2)$ & $3.97(9)$ & \\
C31 & $0.7336(2)$ & $0.3463(2)$ & $-0.1611(1)$ & $3.14(7)$ & \\
\hline
\end{tabular}


Table S-8. Atomic coordinates and $\mathrm{B}_{\text {iso }} / \mathrm{B}_{\text {eq }}$ and occupancy for [trans-(dmpe)2Ru(H)PH(Me)Ph][BPh $]$ (2$\mathrm{BPh}_{4}$ ) (continued).

\begin{tabular}{|c|c|c|c|c|c|}
\hline atom & $\mathbf{x}$ & $\mathbf{y}$ & $\mathbf{z}$ & $B_{\text {eq }}$ & occupancy \\
\hline C32 & $0.7031(2)$ & $0.1526(2)$ & $-0.0597(1)$ & $2.55(7)$ & \\
\hline C33 & $0.7158(2)$ & $0.1279(2)$ & $-0.0003(1)$ & $3.12(7)$ & \\
\hline C34 & $0.7540(2)$ & $0.0397(3)$ & $0.0199(1)$ & $3.75(8)$ & \\
\hline C35 & $0.7818(2)$ & $-0.0282(3)$ & $-0.0190(2)$ & $3.89(9)$ & \\
\hline C36 & $0.7714(2)$ & $-0.0052(3)$ & $-0.0775(2)$ & 3.86(8) & \\
\hline C37 & $0.7324(2)$ & $0.0822(2)$ & $-0.0974(1)$ & $2.91(7)$ & \\
\hline C38 & $0.6753(2)$ & $0.3487(2)$ & $-0.0384(1)$ & $2.50(6)$ & \\
\hline C39 & $0.7570(2)$ & $0.3633(2)$ & $-0.0155(1)$ & $3.23(8)$ & \\
\hline C40 & $0.7815(2)$ & $0.4461(3)$ & $0.0191(2)$ & 3.90(9) & \\
\hline C41 & $0.7256(2)$ & $0.5178(3)$ & $0.0322(1)$ & $3.77(9)$ & \\
\hline C42 & $0.6452(2)$ & $0.5062(2)$ & $0.0107(1)$ & $3.34(8)$ & \\
\hline C43 & $0.6206(2)$ & $0.4230(2)$ & $-0.0234(1)$ & $2.75(7)$ & \\
\hline C44 & $0.8392(4)$ & $0.6959(4)$ & $-0.0639(3)$ & $8.6(2)^{\prime}$ & \\
\hline C45 & $0.8355(4)$ & $0.6099(4)$ & $-0.1021(2)$ & $7.0(1)$ & \\
\hline C46 & $0.9217(6)$ & $0.5731(6)$ & $-0.0991(4)$ & $12.4(3)$ & \\
\hline C47 & $0.9645(5)$ & $0.635(1)$ & $-0.0546(5)$ & $17.3(5)$ & \\
\hline C48 & $1.0196(5)$ & $-0.0864(6)$ & $0.0107(3)$ & 12.1(3) & \\
\hline C49 & $1.0094(4)$ & $-0.0357(6)$ & $-0.0463(3)$ & $9.3(2)$ & \\
\hline B1 & $0.6497(2)$ & $0.2528(2)$ & $-0.0820(1)$ & $2.45(5)$ & \\
\hline
\end{tabular}




\begin{tabular}{|c|c|c|c|c|c|c|}
\hline atom & $U_{11}$ & $\mathbf{U}_{22}$ & $\mathbf{U}_{33}$ & $U_{12}$ & $U_{13}$ & $\mathbf{U}_{23}$ \\
\hline Ru1 & $0.0263(1)$ & $0.0233(1)$ & $0.0277(1)$ & $-0.0012(1)$ & $0.00192(9)$ & $0.00027(10)$ \\
\hline $\mathrm{P} 1$ & $0.0289(4)$ & $0.0277(4)$ & $0.0382(4)$ & $-0.0028(3)$ & $0.0034(3)$ & $0.0008(3)$ \\
\hline P2 & $0.0377(5)$ & $0.0471(5)$ & $0.0296(4)$ & $-0.0066(4)$ & $-0.0000(3)$ & $0.0013(4)$ \\
\hline P3 & $0.0348(5)$ & $0.0279(4)$ & $0.0508(5)$ & $0.0021(3)$ & $0.0122(4)$ & $-0.0009(4)$ \\
\hline $\mathrm{P} 4$ & $0.0449(5)$ & $0.0566(6)$ & $0.0323(5)$ & $-0.0079(4)$ & $-0.0042(4)$ & $0.0066(4)$ \\
\hline P5 & $0.0398(5)$ & $0.0256(4)$ & $0.0645(6)$ & $0.0009(4)$ & $0.0094(4)$ & $0.0006(4)$ \\
\hline $\mathrm{O} 1$ & $0.112(4)$ & $0.239(6)$ & $0.140(4)$ & $-0.043(4)$ & $-0.011(3)$ & $-0.065(4)$ \\
\hline $\mathrm{O} 2$ & $0.132(7)$ & $0.127(7)$ & $0.090(5)$ & $-0.007(5)$ & $0.032(5)$ & $0.054(5)$ \\
\hline C1 & $0.044(2)$ & $0.041(2)$ & $0.084(3)$ & $-0.004(2)$ & $0.008(2)$ & $0.012(2)$ \\
\hline $\mathrm{C} 2$ & $0.029(2)$ & $0.025(2)$ & $0.035(2)$ & $-0.004(1)$ & $0.004(1)$ & $0.003(1)$ \\
\hline C3 & $0.035(2)$ & $0.048(2)$ & $0.042(2)$ & $-0.000(2)$ & $0.003(2)$ & $0.003(2)$ \\
\hline $\mathrm{C} 4$ & $0.045(2)$ & $0.062(2)$ & $0.042(2)$ & $0.006(2)$ & $0.012(2)$ & $0.002(2)$ \\
\hline C5 & $0.036(2)$ & $0.048(2)$ & $0.060(2)$ & $0.009(1)$ & $0.011(2)$ & $0.005(2)$ \\
\hline C6 & $0.037(2)$ & $0.040(2)$ & $0.052(2)$ & $0.006(1)$ & $-0.002(2)$ & $0.007(1)$ \\
\hline C7 & $0.039(2)$ & $0.032(2)$ & $0.038(2)$ & $-0.001(1)$ & $0.005(1)$ & $0.002(1)$ \\
\hline C8 & $0.053(3)$ & $0.083(3)$ & $0.058(3)$ & $0.009(2)$ & $-0.010(2)$ & $0.019(2)$ \\
\hline C9 & $0.055(3)$ & $0.056(2)$ & $0.088(3)$ & $0.023(2)$ & $-0.001(2)$ & $0.022(2)$ \\
\hline C10 & $0.069(3)$ & $0.101(3)$ & $0.035(2)$ & $-0.021(2)$ & $0.008(2)$ & $-0.002(2)$ \\
\hline C11 & $0.085(3)$ & $0.084(3)$ & $0.054(3)$ & $-0.039(3)$ & $-0.014(2)$ & $-0.005(2)$ \\
\hline C12 & $0.065(3)$ & $0.035(2)$ & $0.114(3)$ & $-0.003(2)$ & $0.026(3)$ & $-0.024(2)$ \\
\hline C13 & $0.058(3)$ & $0.045(2)$ & $0.117(4)$ & $0.009(2)$ & $0.048(3)$ & $0.010(2)$ \\
\hline C14 & $0.094(4)$ & $0.065(3)$ & $0.068(3)$ & $-0.006(3)$ & $-0.031(3)$ & $0.033(2)$ \\
\hline C15 & $0.052(3)$ & $0.046(2)$ & $0.107(4)$ & $0.013(2)$ & $-0.006(2)$ & $0.024(2)$ \\
\hline C16 & $0.052(2)$ & $0.093(3)$ & $0.039(2)$ & $-0.013(2)$ & $-0.007(2)$ & $-0.008(2)$ \\
\hline C17 & $0.061(3)$ & $0.132(4)$ & $0.035(2)$ & $-0.029(3)$ & $0.004(2)$ & $0.017(2)$ \\
\hline C18 & $0.058(3)$ & $0.028(2)$ & $0.118(3)$ & $-0.005(2)$ & $0.012(2)$ & $-0.002(2)$ \\
\hline C19 & $0.077(3)$ & $0.039(2)$ & $0.123(4)$ & $0.001(2)$ & $0.050(3)$ & $-0.020(2)$ \\
\hline C20 & $0.036(2)$ & $0.030(2)$ & $0.024(1)$ & $-0.001(1)$ & $0.001(1)$ & $-0.004(1)$ \\
\hline $\mathrm{C} 21$ & $0.041(2)$ & $0.033(2)$ & $0.034(2)$ & $-0.003(1)$ & $-0.002(1)$ & $-0.002(1)$ \\
\hline C22 & $0.040(2)$ & $0.044(2)$ & $0.035(2)$ & $0.005(1)$ & $-0.002(2)$ & $-0.006(1)$ \\
\hline $\mathrm{C} 23$ & $0.036(2)$ & $0.059(2)$ & $0.042(2)$ & $-0.005(2)$ & $0.001(2)$ & $-0.011(2)$ \\
\hline C24 & $0.045(2)$ & $0.044(2)$ & $0.038(2)$ & $-0.010(2)$ & $0.003(2)$ & $0.001(1)$ \\
\hline C25 & $0.037(2)$ & $0.033(2)$ & $0.031(2)$ & $0.002(1)$ & $0.002(1)$ & $-0.001(1)$ \\
\hline C26 & $0.039(2)$ & $0.030(1)$ & $0.031(2)$ & $0.008(1)$ & $0.008(1)$ & $0.001(1)$ \\
\hline C27 & $0.048(2)$ & $0.052(2)$ & $0.035(2)$ & $-0.000(2)$ & $0.004(2)$ & $-0.003(2)$ \\
\hline C28 & $0.064(3)$ & $0.074(3)$ & $0.030(2)$ & $0.021(2)$ & $0.003(2)$ & $-0.000(2)$ \\
\hline C29 & $0.069(3)$ & $0.066(3)$ & $0.042(2)$ & $0.028(2)$ & $0.024(2)$ & $0.021(2)$ \\
\hline C30 & $0.056(2)$ & $0.043(2)$ & $0.056(2)$ & $0.013(2)$ & $0.027(2)$ & $0.012(2)$ \\
\hline C31 & $0.042(2)$ & $0.036(2)$ & $0.043(2)$ & $0.007(1)$ & $0.014(2)$ & $0.002(1)$ \\
\hline
\end{tabular}


Table S-10. Anisotropic Displacement Parameters for [trans-(dmpe)2Ru(H)PH(Me)Ph][BPh $]\left(2-\mathrm{BPh}_{4}\right)$ (continued).

\begin{tabular}{llllrrr} 
atom & $\mathbf{U}_{11}$ & $\mathbf{U}_{\mathbf{2 2}}$ & $\mathbf{U}_{33}$ & $\mathbf{U}_{\mathbf{1 2}}$ & $\mathbf{U}_{13}$ & \multicolumn{1}{c}{$\mathbf{U}_{\mathbf{2 3}}$} \\
\hline C32 & $0.033(2)$ & $0.033(2)$ & $0.032(2)$ & $-0.001(1)$ & $0.005(1)$ & $0.002(1)$ \\
C33 & $0.044(2)$ & $0.041(2)$ & $0.034(2)$ & $0.009(1)$ & $0.006(1)$ & $-0.000(1)$ \\
C34 & $0.051(2)$ & $0.050(2)$ & $0.040(2)$ & $0.006(2)$ & $0.000(2)$ & $0.009(2)$ \\
C35 & $0.050(2)$ & $0.037(2)$ & $0.059(2)$ & $0.013(2)$ & $-0.001(2)$ & $0.005(2)$ \\
C36 & $0.053(2)$ & $0.043(2)$ & $0.050(2)$ & $0.012(2)$ & $-0.000(2)$ & $-0.012(2)$ \\
C37 & $0.036(2)$ & $0.037(2)$ & $0.037(2)$ & $0.001(1)$ & $0.005(1)$ & $-0.005(1)$ \\
C38 & $0.038(2)$ & $0.031(2)$ & $0.026(2)$ & $0.000(1)$ & $0.003(1)$ & $0.004(1)$ \\
C39 & $0.042(2)$ & $0.041(2)$ & $0.040(2)$ & $0.001(1)$ & $0.003(2)$ & $-0.003(1)$ \\
C40 & $0.046(2)$ & $0.050(2)$ & $0.049(2)$ & $-0.010(2)$ & $-0.009(2)$ & $0.001(2)$ \\
C41 & $0.065(3)$ & $0.039(2)$ & $0.038(2)$ & $-0.011(2)$ & $0.001(2)$ & $-0.007(1)$ \\
C42 & $0.061(2)$ & $0.032(2)$ & $0.034(2)$ & $0.003(2)$ & $0.007(2)$ & $-0.005(1)$ \\
C43 & $0.040(2)$ & $0.036(2)$ & $0.029(2)$ & $-0.001(1)$ & $0.004(1)$ & $-0.001(1)$ \\
C44 & $0.109(5)$ & $0.078(4)$ & $0.136(5)$ & $0.009(3)$ & $-0.012(4)$ & $-0.025(4)$ \\
C45 & $0.110(5)$ & $0.079(3)$ & $0.077(3)$ & $-0.004(3)$ & $0.010(3)$ & $0.009(3)$ \\
C46 & $0.175(9)$ & $0.137(6)$ & $0.166(7)$ & $0.099(6)$ & $0.045(6)$ & $0.010(5)$ \\
C47 & $0.058(5)$ & $0.36(2)$ & $0.23(1)$ & $0.015(7)$ & $0.000(6)$ & $-0.00(1)$ \\
C48 & $0.155(7)$ & $0.219(9)$ & $0.081(4)$ & $-0.112(6)$ & $-0.005(4)$ & $0.048(5)$ \\
C49 & $0.086(4)$ & $0.164(6)$ & $0.102(5)$ & $-0.022(4)$ & $0.009(3)$ & $0.040(5)$ \\
\hline
\end{tabular}

The general temperature factor expression:

$\exp \left(-2 \pi^{2}\left(a^{* 2} U_{11} h^{2}+b^{\star 2} U_{22} k^{2}+c^{\star 2} U_{33} l^{2}+2 a^{*} b^{*} U_{12} h k+2 a^{*} c^{*} U_{13} h l+2 b^{*} c^{*} U_{23} k l\right)\right)$ 
Table S-11. Bond Lengths $(\AA)$ for $\left[\right.$ trans- $\left.(\mathrm{dmpe}) 2 \mathrm{Ru}(\mathrm{H}) \mathrm{PH}(\mathrm{Me}) \mathrm{Ph}^{-} \mathrm{BPh}_{4}\right]\left(\mathbf{2}-\mathrm{BPh}_{4}\right)$.

\begin{tabular}{llllll} 
atom & atom & distance & atom & atom & distance \\
\hline Ru1 & P1 & $2.3423(8)$ & Ru1 & P2 & $2.3173(8)$ \\
Ru1 & P3 & $2.3158(8)$ & Ru1 & P4 & $2.3118(9)$ \\
Ru1 & P5 & $2.3219(8)$ & P1 & C1 & $1.847(4)$ \\
P1 & C2 & $1.825(3)$ & P2 & C8 & $1.826(4)$ \\
P2 & C10 & $1.815(4)$ & P2 & C11 & $1.812(4)$ \\
P3 & C9 & $1.836(4)$ & P3 & C12 & $1.814(4)$ \\
P3 & C13 & $1.816(4)$ & P4 & C14 & $1.834(4)$ \\
P4 & C16 & $1.827(4)$ & P4 & C17 & $1.824(4)$ \\
P5 & C15 & $1.844(4)$ & P5 & C18 & $1.823(4)$ \\
P5 & C19 & $1.812(4)$ & O1 & C44 & $1.357(7)$ \\
O1 & C47 & $1.47(1)$ & O2 & C48 & $1.25(1)$ \\
O2 & C49 & $1.356(10)$ & C2 & C3 & $1.386(4)$ \\
C2 & C7 & $1.399(4)$ & C3 & C4 & $1.383(5)$ \\
C4 & C5 & $1.387(5)$ & C5 & C6 & $1.376(5)$ \\
C6 & C7 & $1.381(5)$ & C8 & C9 & $1.493(6)$ \\
C14 & C15 & $1.483(6)$ & C20 & C21 & $1.414(4)$ \\
C20 & C25 & $1.391(4)$ & C20 & B1 & $1.653(4)$ \\
C21 & C22 & $1.369(4)$ & C22 & C23 & $1.383(5)$ \\
C23 & C24 & $1.375(5)$ & C26 & C25 & $1.394(4)$ \\
C26 & C27 & $1.409(4)$ & C27 & C28 & $1.396(4)$ \\
C26 & B1 & $1.645(4)$ & C29 & C30 & $1.396(5)$ \\
C28 & C29 & $1.375(6)$ & C32 & C33 & $1.370(5)$ \\
C30 & C31 & $1.386(4)$ & C32 & B1 & $1.401(4)$ \\
C32 & C37 & $1.389(4)$ & C34 & C35 & $1.647(4)$ \\
C33 & C34 & $1.387(4)$ & C36 & C37 & $1.382(5)$ \\
C35 & C36 & $1.373(4)$ & C38 & C43 & $1.397(4)$ \\
C38 & C39 & $1.403(4)$ & C39 & C40 & $1.393(4)$ \\
C38 & B1 & $1.649(4)$ & C41 & C42 & $1.367(5)$ \\
C40 & C41 & $1.373(5)$ & C44 & C45 & $1.440(7)$ \\
C42 & C43 & $1.392(4)$ & C47 & $1.44(1)$ \\
C45 & C46 & $1.486(9)$ & & & \\
C48 & C49 & $1.468(7)$ & & &
\end{tabular}




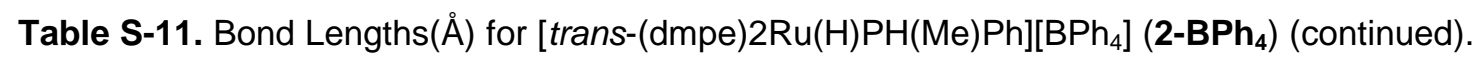

\begin{tabular}{|c|c|c|c|c|c|}
\hline atom & atom & distance & atom & atom & distance \\
\hline Ru1 & $\mathrm{H} 1$ & 1.62 & P1 & $\mathrm{H} 2$ & 1.61 \\
\hline C1 & H3 & 0.95 & C1 & $\mathrm{H} 4$ & 0.95 \\
\hline C1 & H5 & 0.95 & C3 & $\mathrm{H} 6$ & 0.95 \\
\hline C4 & $\mathrm{H} 7$ & 0.95 & C5 & H8 & 0.95 \\
\hline C6 & H9 & 0.95 & C7 & $\mathrm{H} 10$ & 0.95 \\
\hline C8 & $\mathrm{H} 11$ & 0.95 & C8 & $\mathrm{H} 12$ & 0.95 \\
\hline C9 & $\mathrm{H} 13$ & 0.95 & C9 & $\mathrm{H} 14$ & 0.95 \\
\hline C10 & H15 & 0.95 & C10 & $\mathrm{H} 16$ & 0.95 \\
\hline C10 & $\mathrm{H} 17$ & 0.95 & C11 & $\mathrm{H} 18$ & 0.95 \\
\hline C11 & $\mathrm{H} 19$ & 0.95 & C11 & $\mathrm{H} 2 \mathrm{O}$ & 0.95 \\
\hline C12 & H21 & 0.95 & C12 & $\mathrm{H} 22$ & 0.95 \\
\hline C12 & $\mathrm{H} 23$ & 0.95 & C13 & $\mathrm{H} 24$ & 0.95 \\
\hline C13 & H25 & 0.95 & C13 & H26 & 0.95 \\
\hline C14 & $\mathrm{H} 27$ & 0.95 & C14 & H28 & 0.95 \\
\hline C15 & H29 & 0.95 & C15 & H30 & 0.95 \\
\hline C16 & H31 & 0.95 & C16 & H32 & 0.95 \\
\hline C16 & H33 & 0.95 & C17 & H34 & 0.95 \\
\hline C17 & H35 & 0.95 & C17 & H36 & 0.95 \\
\hline C18 & H37 & 0.95 & C18 & H38 & 0.95 \\
\hline C18 & H39 & 0.95 & C19 & $\mathrm{H} 40$ & 0.95 \\
\hline C19 & H41 & 0.95 & C19 & H42 & 0.95 \\
\hline C21 & $\mathrm{H} 43$ & 0.95 & C22 & H44 & 0.95 \\
\hline C23 & $\mathrm{H} 45$ & 0.95 & C24 & $\mathrm{H} 46$ & 0.95 \\
\hline C25 & $\mathrm{H} 47$ & 0.95 & C27 & $\mathrm{H} 48$ & 0.95 \\
\hline C28 & H49 & 0.95 & C29 & H50 & 0.95 \\
\hline C30 & H51 & 0.95 & C31 & H52 & 0.95 \\
\hline C33 & H53 & 0.95 & C34 & H54 & 0.95 \\
\hline C35 & H55 & 0.95 & C36 & H56 & 0.95 \\
\hline C37 & H57 & 0.95 & C39 & H58 & 0.95 \\
\hline C40 & H59 & 0.95 & C41 & $\mathrm{H} 60$ & 0.95 \\
\hline C42 & $\mathrm{H} 61$ & 0.95 & C43 & H62 & 0.95 \\
\hline C44 & H63 & 0.95 & C44 & $\mathrm{H} 64$ & 0.95 \\
\hline C45 & H65 & 0.95 & C45 & H66 & 0.95 \\
\hline C46 & H67 & 0.95 & C46 & H68 & 0.95 \\
\hline C47 & H69 & 0.95 & C47 & $\mathrm{H} 70$ & 0.95 \\
\hline C48 & $\mathrm{H} 71$ & 0.94 & C48 & H72 & 0.95 \\
\hline C49 & H73 & 0.95 & C49 & H74 & 0.95 \\
\hline
\end{tabular}


Table S-12. Bond Angles $\left({ }^{\circ}\right)$ for $[$ trans-(dmpe)2Ru(H)PH(Me)Ph][BPh $]\left(2-\mathrm{BPh}_{4}\right)$.

\begin{tabular}{|c|c|c|c|c|c|c|c|}
\hline atom & atom & atom & angle & atom & atom & atom & angle \\
\hline P1 & Ru1 & P2 & $99.23(3)$ & P1 & Ru1 & P3 & 91.91(3) \\
\hline P1 & Ru1 & P4 & $96.34(3)$ & P1 & Ru1 & P5 & $99.84(3)$ \\
\hline P2 & Ru1 & P3 & $83.82(3)$ & P2 & Ru1 & P4 & $164.43(3)$ \\
\hline P2 & Ru1 & P5 & $94.04(3)$ & P3 & Ru1 & P4 & $95.24(3)$ \\
\hline P3 & Ru1 & P5 & $168.25(3)$ & P4 & Ru1 & P5 & $83.71(3)$ \\
\hline Ru1 & P1 & C1 & $121.6(1)$ & Ru1 & P1 & $\mathrm{C} 2$ & $123.85(9)$ \\
\hline C1 & P1 & $\mathrm{C} 2$ & $99.0(2)$ & Ru1 & P2 & C8 & 108.2(1) \\
\hline Ru1 & P2 & C10 & $122.7(1)$ & Ru1 & P2 & C11 & 119.1(1) \\
\hline C8 & P2 & C10 & $103.1(2)$ & C8 & P2 & C11 & 100.3(2) \\
\hline C10 & P2 & C11 & $100.3(2)$ & Ru1 & P3 & C9 & 108.2(1) \\
\hline Ru1 & P3 & C12 & $121.3(1)$ & Ru1 & P3 & C13 & $119.5(1)$ \\
\hline C9 & P3 & C12 & $103.7(2)$ & C9 & P3 & C13 & $101.4(2)$ \\
\hline C12 & P3 & C13 & $100.1(2)$ & Ru1 & P4 & C14 & $108.8(1)$ \\
\hline Ru1 & P4 & C16 & $122.4(1)$ & Ru1 & P4 & C17 & $119.2(1)$ \\
\hline C14 & P4 & C16 & $102.1(2)$ & C14 & P4 & C17 & $100.5(2)$ \\
\hline C16 & P4 & C17 & $100.6(2)$ & Ru1 & P5 & C15 & $107.4(1)$ \\
\hline Ru1 & P5 & C18 & $117.2(1)$ & Ru1 & P5 & C19 & $124.4(1)$ \\
\hline C15 & P5 & C18 & $101.0(2)$ & C15 & P5 & C19 & $102.7(2)$ \\
\hline C18 & P5 & C19 & $101.0(2)$ & C44 & O1 & C47 & $101.9(6)$ \\
\hline C48 & $\mathrm{O} 2$ & C49 & $93.5(6)$ & P1 & C2 & C3 & $121.6(2)$ \\
\hline P1 & C2 & C7 & 121.2(2) & C3 & $\mathrm{C} 2$ & C7 & $117.2(3)$ \\
\hline C2 & C3 & C4 & $121.2(3)$ & C3 & C4 & C5 & $120.3(3)$ \\
\hline C4 & C5 & C6 & 119.6(3) & C5 & C6 & C7 & 119.6(3) \\
\hline C2 & C7 & C6 & $122.0(3)$ & P2 & C8 & C9 & $110.9(3)$ \\
\hline P3 & C9 & C8 & $109.0(2)$ & P4 & C14 & C15 & $110.7(3)$ \\
\hline P5 & C15 & C14 & $109.1(3)$ & C21 & C20 & C25 & $113.9(3)$ \\
\hline C21 & C20 & B1 & $122.2(3)$ & C25 & C20 & B1 & $123.9(3)$ \\
\hline C20 & C21 & C22 & $123.6(3)$ & C21 & C22 & C23 & $120.2(3)$ \\
\hline C22 & C23 & C24 & $118.9(3)$ & C23 & C24 & C25 & $119.9(3)$ \\
\hline C20 & C25 & C24 & 123.5(3) & C27 & C26 & C31 & $114.3(3)$ \\
\hline C27 & C26 & B1 & $122.0(3)$ & C31 & C26 & B1 & 123.7(3) \\
\hline C26 & C27 & C28 & $122.3(3)$ & C27 & C28 & C29 & 120.5(3) \\
\hline C28 & C29 & C30 & $119.0(3)$ & C29 & C30 & C31 & $120.1(3)$ \\
\hline C26 & C31 & C30 & 123.7(3) & C33 & C32 & C37 & $114.9(3)$ \\
\hline C33 & C32 & B1 & $121.2(3)$ & C37 & C32 & B1 & $123.7(3)$ \\
\hline C32 & C33 & C34 & $122.9(3)$ & C33 & C34 & C35 & $120.2(3)$ \\
\hline C34 & C35 & C36 & 118.2(3) & C35 & C36 & C37 & 121.3(3) \\
\hline C32 & C37 & C36 & $122.4(3)$ & C39 & C38 & C43 & 114.7(3) \\
\hline C39 & C38 & B1 & $120.8(3)$ & C43 & C38 & B1 & $124.4(3)$ \\
\hline
\end{tabular}


Table S-12. Bond Angles $\left({ }^{\circ}\right)$ for [trans-(dmpe)2Ru(H)PH(Me)Ph][BPh $]$ (2-BPh 4$)$ (continued).

\begin{tabular}{llllllll} 
atom & atom & atom & angle & atom & atom & atom & angle \\
\hline C38 & C39 & C40 & $122.3(3)$ & C39 & C40 & C41 & $120.8(3)$ \\
C40 & C41 & C42 & $118.7(3)$ & C41 & C42 & C43 & $120.6(3)$ \\
C38 & C43 & C42 & $122.9(3)$ & O1 & C44 & C45 & $109.9(5)$ \\
C44 & C45 & C46 & $104.3(5)$ & C45 & C46 & C47 & $103.9(6)$ \\
O1 & C47 & C46 & $108.4(7)$ & O2 & C48 & C49 & $128.4(10)$ \\
O2 & C49 & C48 & $126.9(8)$ & C20 & B1 & C26 & $109.6(2)$ \\
C20 & B1 & C32 & $108.2(2)$ & C20 & B1 & C38 & $110.9(2)$ \\
C26 & B1 & C32 & $109.8(2)$ & C26 & B1 & C38 & $108.6(2)$ \\
C32 & B1 & C38 & $109.9(2)$ & & & & \\
\hline
\end{tabular}


Table S-13. Torsion Angles $\left({ }^{\circ}\right)$ for $\left[\right.$ trans-(dmpe)2Ru(H)PH(Me)Ph] $\left[\mathrm{BPh}_{4}\right]\left(\mathbf{2}-\mathrm{BPh}_{4}\right)$.

\begin{tabular}{|c|c|c|c|c|c|c|c|c|c|}
\hline om & tom & to & 10! & & 0 & to & aton & ato & \\
\hline Ru1 & $\mathrm{P} 1$ & $\mathrm{C} 2$ & C3 & $90.2(3)$ & Ru1 & $\mathrm{P} 1$ & $\mathrm{C} 2$ & C7 & $-89.2(2)$ \\
\hline Ru1 & P2 & C8 & C9 & 37.1(3) & Ru1 & P3 & C9 & C8 & $40.0(3)$ \\
\hline Ru1 & P4 & C14 & C15 & $34.9(3)$ & Ru1 & P5 & C15 & C14 & $42.6(3)$ \\
\hline P1 & Ru1 & P2 & C8 & $-100.2(1)$ & P1 & Ru1 & P2 & C10 & $19.4(2)$ \\
\hline P1 & Ru1 & P2 & C11 & $146.3(2)$ & P1 & Ru1 & P3 & C9 & $85.4(1)$ \\
\hline P1 & Ru1 & P3 & C12 & $-34.0(2)$ & P1 & Ru1 & P3 & C13 & $-159.4(2)$ \\
\hline P1 & Ru1 & P4 & C14 & $-105.7(2)$ & P1 & Ru1 & P4 & C16 & 12.9(2) \\
\hline P1 & Ru1 & P4 & C17 & $140.1(2)$ & P1 & Ru1 & P5 & C15 & $78.9(2)$ \\
\hline P1 & Ru1 & P5 & C18 & $-168.4(2)$ & P1 & Ru1 & P5 & C19 & $-40.6(2)$ \\
\hline P1 & C2 & C3 & C4 & $-178.6(3)$ & P1 & C2 & C7 & C6 & $178.8(2)$ \\
\hline P2 & Ru1 & P1 & C1 & $39.0(2)$ & P2 & Ru1 & P1 & $\mathrm{C} 2$ & $-90.3(1)$ \\
\hline P2 & Ru1 & P3 & C9 & $-13.7(1)$ & P2 & Ru1 & P3 & C12 & $-133.1(2)$ \\
\hline P2 & Ru1 & P3 & C13 & $101.5(2)$ & P2 & Ru1 & P4 & C14 & $76.0(2)$ \\
\hline P2 & Ru1 & P4 & C16 & $-165.4(2)$ & P2 & Ru1 & P4 & C17 & $-38.2(2)$ \\
\hline P2 & Ru1 & P5 & C15 & $0(2)$ & P2 & Ru1 & P5 & C18 & $-68.3(2)$ \\
\hline P2 & Ru1 & P5 & C19 & & P2 & C8 & C9 & P3 & $-49.3(4)$ \\
\hline P3 & Ru1 & P1 & C1 & $-45.0(2)$ & P3 & Ru1 & P1 & $\mathrm{C} 2$ & $-174.4(1)$ \\
\hline P3 & Ru1 & P2 & C8 & $-9.3(1)$ & P3 & Ru1 & P2 & C10 & 110.3(2) \\
\hline P3 & Ru1 & P2 & C11 & $-122.8(2)$ & P3 & Ru1 & P4 & C14 & 161.8(2) \\
\hline P3 & Ru1 & P4 & C16 & $-79.6(2)$ & P3 & Ru1 & P4 & C17 & $47.5(2)$ \\
\hline P3 & Ru1 & P5 & C15 & $-101.9(2)$ & P3 & Ru1 & P5 & C18 & $10.7(3)$ \\
\hline P3 & Ru1 & P5 & C19 & $5(2)$ & P4 & Ru1 & P1 & C1 & $-140.5(2)$ \\
\hline P4 & u1 & P1 & C2 & & P4 & Ru1 & P2 & C8 & $.1(2)$ \\
\hline P4 & Ru1 & P2 & C10 & -162 & P4 & Ru1 & P2 & C11 & $-35.4(2)$ \\
\hline P4 & Ru1 & P3 & C9 & $-178.1(1)$ & P4 & Ru1 & P3 & C12 & $62.5(2)$ \\
\hline P4 & Ru1 & P3 & C13 & $-62.9(2)$ & P4 & Ru1 & P5 & C15 & $-16.4(2)$ \\
\hline P4 & Ru1 & P5 & C18 & $96.2(2)$ & P4 & Ru1 & P5 & C19 & $-136.0(2)$ \\
\hline P4 & C14 & C15 & P5 & $-49.5(3)$ & P5 & Ru1 & P1 & C1 & $134.8(2)$ \\
\hline P5 & Ru1 & P1 & C2 & $5.4(1)$ & P5 & Ru1 & P2 & C8 & 159.1(1) \\
\hline P5 & Ru1 & P2 & C10 & -81 & P5 & Ru1 & P2 & C1 & $45.6(2)$ \\
\hline P5 & Ru1 & P3 & C9 & & P5 & Ru1 & P3 & C12 & $146.8(2)$ \\
\hline P5 & Ru1 & P3 & C13 & & P5 & Ru1 & P4 & C14 & $-6.4(2)$ \\
\hline P5 & Ru1 & P4 & C16 & $112.1(2)$ & P5 & Ru1 & P4 & C17 & $-120.7(2)$ \\
\hline 01 & C44 & C45 & C46 & $-26.4(7)$ & 01 & C47 & C46 & C45 & $14(1)$ \\
\hline C1 & P1 & C2 & C3 & $-48.0(3)$ & C1 & P1 & C2 & C7 & $132.6(3)$ \\
\hline C2 & C3 & C4 & C5 & $-0.7(5)$ & $\mathrm{C} 2$ & C7 & C6 & C5 & $0.2(5)$ \\
\hline C3 & C2 & C7 & C6 & $-0.6(4)$ & C3 & C4 & C5 & C6 & $0.2(5)$ \\
\hline C4 & C3 & C2 & C7 & $0.9(5)$ & C4 & C5 & C6 & C7 & $0.1(5)$ \\
\hline C8 & C9 & P3 & C12 & 170.0(3) & C8 & C9 & P3 & C13 & $-86.5(3)$ \\
\hline
\end{tabular}


Table S-13. Torsion Angles $\left({ }^{\circ}\right)$ for [trans-(dmpe)2Ru(H)PH(Me)Ph][BPh $]$ (2-BPh ${ }_{4}$ (continued).

\begin{tabular}{|c|c|c|c|c|c|c|c|c|c|}
\hline om & & & & & Q & to & to & ton & \\
\hline C9 & C8 & P2 & C10 & $-94.2(3)$ & $\mathrm{C9}$ & C8 & P2 & C11 & $162.5(3)$ \\
\hline C14 & C15 & P5 & C18 & & C14 & C15 & P5 & C19 & \\
\hline C15 & C14 & $\mathrm{P} 4$ & C16 & $-95.8(3)$ & C15 & C14 & $\mathrm{P} 4$ & C17 & $160.9(3)$ \\
\hline C20 & C21 & C22 & C23 & $-0.4(5)$ & C20 & C25 & C24 & C23 & $0.2(5)$ \\
\hline C20 & B1 & $\mathrm{C} 26$ & $\mathrm{C} 27$ & $-30.1(4)$ & $\mathrm{C} 20$ & $\mathrm{~B} 1$ & $\mathrm{C} 26$ & C31 & $152.9(3)$ \\
\hline C20 & B1 & C32 & C33 & -78.5 & C20 & B1 & C32 & C37 & $95.6(3)$ \\
\hline C20 & B1 & C38 & C39 & 157.5 & $\mathrm{C} 20$ & B1 & $\mathrm{C} 38$ & $\mathrm{C} 43$ & $-25.5(4)$ \\
\hline C21 & C20 & C25 & C24 & -1.7 & C21 & C20 & B1 & C26 & -50 \\
\hline $\mathrm{C} 21$ & $\mathrm{C} 20$ & B1 & $\mathrm{C} 32$ & -170 & $\mathrm{C} 21$ & $\mathrm{C} 20$ & & $\mathrm{C} 38$ & \\
\hline C21 & C22 & C23 & C24 & -1.3 & C22 & C21 & C20 & C25 & $1.8(4)$ \\
\hline C22 & $\mathrm{C} 21$ & $\mathrm{C} 20$ & B1 & -176.4 & $\mathrm{C} 22$ & $\mathrm{C} 23$ & $\mathrm{C} 24$ & $\mathrm{C} 25$ & \\
\hline C24 & $\mathrm{C} 25$ & $\mathrm{C} 20$ & B1 & $176.5(3)$ & $\mathrm{C} 25$ & $\mathrm{C} 20$ & B1 & C26 & $131.1(3)$ \\
\hline C25 & C20 & B1 & C32 & & $\mathrm{C} 25$ & $\mathrm{C} 20$ & B1 & C38 & $-109.1(3)$ \\
\hline C26 & C27 & C28 & C29 & & C26 & C31 & C30 & C29 & $0.2(5)$ \\
\hline C26 & B1 & C3 & $\mathrm{C} 3$ & & $\mathrm{C} 26$ & B1 & C32 & $\mathrm{C} 37$ & -23. \\
\hline C26 & B1 & C38 & C39 & -82 & C26 & B1 & C38 & C43 & (3) \\
\hline C27 & $\mathrm{C} 26$ & C31 & $\mathrm{C} 30$ & & $\mathrm{C} 27$ & $\mathrm{C} 26$ & B1 & C32 & (3) \\
\hline C27 & C26 & B1 & C38 & -151. & C27 & C28 & C29 & C30 & $0.0(6)$ \\
\hline C28 & $\mathrm{C} 27$ & C26 & C31 & & $\mathrm{C} 28$ & $\mathrm{C} 27$ & $\mathrm{C} 26$ & B1 & $-177.7(3)$ \\
\hline C28 & C29 & C30 & C31 & -0.3 & C30 & C31 & $\mathrm{C} 26$ & B1 & $177.4(3)$ \\
\hline C31 & $\mathrm{C} 26$ & B1 & C32 & -88 & C31 & $\mathrm{C} 26$ & B1 & C38 & $31.7(4)$ \\
\hline C32 & C33 & C34 & C35 & & C32 & C37 & C36 & C35 & $1.4(6)$ \\
\hline C32 & B1 & $\mathrm{C} 3$ & C3 & & C32 & B1 & C38 & $\mathrm{C} 43$ & $-145.0(3)$ \\
\hline C33 & C32 & C37 & C36 & & C33 & C32 & B1 & C38 & $42.7(4)$ \\
\hline C33 & C34 & C35 & C36 & & C34 & C33 & C32 & C37 & $-0.5(5)$ \\
\hline C34 & C33 & C32 & B1 & $174.1(3)$ & C34 & C35 & C36 & C37 & $-1.6(6)$ \\
\hline C36 & C37 & C32 & $\mathrm{B} 1$ & $-174.7(3)$ & C37 & C32 & $\mathrm{B} 1$ & C38 & $-143.3(3)$ \\
\hline C38 & C39 & $\mathrm{C} 40$ & C41 & $-0.3(5)$ & C38 & C43 & $\mathrm{C} 42$ & C41 & $-1.0(5)$ \\
\hline C39 & C38 & C43 & C42 & & C39 & C40 & C41 & C42 & $0.4(5)$ \\
\hline $\mathrm{C} 40$ & C39 & C38 & C43 & $-0.4(4)$ & $\mathrm{C} 40$ & C39 & C38 & B1 & $176.8(3)$ \\
\hline C40 & C41 & C42 & C43 & & C42 & C43 & C38 & B1 & $-176.1(3)$ \\
\hline C44 & 01 & C47 & C46 & $-30(1)$ & C44 & C45 & C46 & C47 & $5.9(9)$ \\
\hline C45 & C44 & 01 & C47 & $34.6(8)$ & & & & & \\
\hline
\end{tabular}


Table S-14. Non-bonded Contacts out to $3.75 \AA$ for [trans-(dmpe)2Ru(H)PH(Me)Ph][BPh $]\left(2-\mathrm{BPh}_{4}\right)$.

\begin{tabular}{llllllll} 
atom & atom & distance & ADC & atom & atom & distance & ADC \\
\hline O1 & C48 & $3.607(9)$ & 56501 & O1 & C17 & $3.715(6)$ & 65502 \\
O1 & C16 & $3.738(6)$ & 65502 & O2 & C17 & $3.243(9)$ & 55404 \\
O2 & C16 & $3.249(9)$ & 55404 & O2 & C35 & $3.668(9)$ & 75503 \\
C3 & C40 & $3.599(5)$ & 1 & C4 & C40 & $3.687(5)$ & 1 \\
C5 & C12 & $3.628(5)$ & 64502 & C6 & C22 & $3.700(5)$ & 4 \\
C7 & C22 & $3.716(4)$ & 4 & C8 & C45 & $3.641(7)$ & 66503 \\
C9 & C31 & $3.704(5)$ & 66503 & C10 & C43 & $3.417(5)$ & 1 \\
C10 & C25 & $3.599(5)$ & 1 & C11 & C36 & $3.537(6)$ & 65503 \\
C13 & C30 & $3.629(5)$ & 45504 & C13 & C37 & $3.636(5)$ & 45504 \\
C13 & C29 & $3.666(6)$ & 45504 & C17 & C48 & $3.593(8)$ & 65502 \\
C35 & C48 & $3.576(7)$ & 75503 & C35 & C49 & $3.695(7)$ & 75503 \\
C40 & C45 & $3.708(6)$ & 1 & & & & \\
\hline
\end{tabular}




\section{References.}

${ }^{1}$ Kaplan, A.W.; Ritter, J.C.M.; Bergman, R.G. J. Am. Chem. Soc. 1998, 120, 6828-6829.

${ }^{2}$ Ashworth, T.V.; Singleton, E.; Hough, J.J. J. Chem. Soc. Dalton. 1977, 19, 1809-1815.

${ }^{3}$ Hallman, P.S.; Mcgarvey, B.R.; Wilkinson, G. J. Chem. Soc. A 1968, 3143.

${ }^{4}$ No nitrogen from the $\mathrm{N}_{2}$ ligand was detected by elemental analysis.

${ }^{5}$ Moncarz, J.R.; Brunker, T. J.; Jewett, J.C.; Orchowski, M.; Glueck, D.S.; Sommer, R.D.; Lam, K.C.; Incarvito, C.D.; Concolino, T. E.; Ceccarelli, C.; Zakharov, L. N.; Rheingold, A. L. Organometallics, 2003, 22, 3205-3221.

${ }^{6}$ SMART: Area-Detector Software Package, Bruker Analytical X-ray Systems, Inc.: Madison, WI, (2001-03)

7 APEX:

${ }^{8}$ SAINT: SAX Area-Dectector Integration Program, V6.40; Bruker Analytical X-ray Systems Inc.: Madison, WI, (2003)

9 XPREP:(v 6.12) Part of the SHELXTL Crystal Structure Determination Package, Bruker Analytical X-ray Systems, Inc.: Madison, WI, (2001)

${ }^{10}$ SADABS: Bruker-Nonius Area Detector Scaling and Absorption v. 2.05 Bruker Analytical Xray Systems, Inc.: Madison, WI (2003).

${ }^{11}$ SIR97: Altomare, A., Burla, M. C., Camalli, M., Cascarano, G., Giacovazzo, C., Guagliardi, A., Moliterni, A. G. G., Polidori, G., \& Spagna, R. SIR97: A new tool for crystal structure determination and refinement. J. App. Cryst. (1998)

${ }^{12}$ DIRDIF94: Beurskens, P.T., Admiraal, G., Beurskens, G., Bosman, W.P., de Gelder, R., Israel, R. and Smits, J.M.M.(1994). The DIRDIF-94 program system, Technical Report of the Crystallography Laboratory, University of Nijmegen, The Netherlands.

${ }^{13}$ Least-Squares: Function minimized $\sum w\left(\left|\mathrm{~F}_{\mathrm{o}}\right|-\left|\mathrm{F}_{\mathrm{c}}\right|\right)^{2}$

${ }^{14}$ Standard deviation of an observation of unit weight:

$$
\begin{aligned}
& {\left[\sum w\left(\left|F_{\mathrm{o}}\right|-\left|\mathrm{F}_{\mathrm{c}}\right|\right)^{2} /\left(\mathrm{N}_{\mathrm{o}}-\mathrm{N}_{\mathrm{v}}\right)\right]^{1 / 2}} \\
& \text { where } \mathrm{N}_{\mathrm{o}}=\text { number of observations } \\
& \qquad \mathrm{N}_{\mathrm{v}}=\text { number of variables }
\end{aligned}
$$

${ }^{15}$ Cromer, D. T. \& Waber, J. T.; "International Tables for X-ray Crystallography", Vol. IV, The Kynoch Press, Birmingham, England, Table 2.2 A (1974).

${ }^{16}$ Ibers, J. A. \& Hamilton, W. C.; Acta Crystallogr., 17, 781 (1964).

${ }^{17}$ Creagh, D. C. \& McAuley, W.J .; "International Tables for Crystallography", Vol C, (A.J.C. Wilson, ed.), Kluwer Academic Publishers, Boston, Table 4.2.6.8, pages 219-222 (1992). 
${ }^{18}$ Creagh, D. C. \& Hubbell, J.H..; "International Tables for Crystallography", Vol C, (A.J.C.

Wilson, ed.), Kluwer Academic Publishers, Boston, Table 4.2.4.3, pages 200-206 (1992).

${ }^{19}$ teXsan: Crystal Structure Analysis Package, Molecular Structure Corporation (1985 \& 1992). 